\title{
Feynman Path Integrals and the Trace Formula for the Schrödinger Operators
}

\author{
S. Albeverio ${ }^{2}$, Ph. Blanchard ${ }^{1}$, and R. Hoegh-Krohn ${ }^{3 \star}$ \\ 1 Fakultät für Physik, Universität Bielefeld, Bielefeld Federal Republic of Germany \\ 2 Fakultät für Mathematik, Ruhr-Universität Bochum, Bochum, Federal Republic of Germany \\ 3 Matematisk Institutt, Universitetet i Oslo, Oslo, Norway
}

\begin{abstract}
We study Schrödinger operators of the form $H=-\frac{h^{2}}{2} \Delta+\frac{1}{2} x \cdot A^{2} x$ $+V(x)$ on $\mathbb{R}^{d}$, where $A^{2}$ is a strictly positive symmetric $d \times d$ matrix and $V(x)$ is a continuous real function which is the Fourier transform of a bounded measure. If $\lambda_{n}$ are the eigenvalues of $H$ we show that the theta function $\theta(t)=\sum_{n} \exp \left(-\frac{i}{h} t \lambda_{n}\right)$ is explicitly expressible in terms of infinite dimensional oscillatory integrals (Feynman path integrals) over the Hilbert space of closed trajectories. We use these explicit expressions to give the asymptotic behaviour of $\theta(t)$ for small $h$ in terms of classical periodic orbits, thus obtaining a trace formula for the Schrödinger operators. This then yields an asymptotic expansion of the spectrum of $H$ in terms of the periodic orbits of the corresponding classical mechanical system. These results extend to the physical case the recent work on Poisson and trace formulae for compact manifolds.
\end{abstract}

\section{Introduction}

The study of the relations of quantum mechanics and classical mechanics goes back to the very origin of quantum physics, i.e. to the years where the "old quantum theory" of Bohr, Einstein, Sommerfeld was developed and was to lead to what is now understood as quantum mechanics (Heisenberg, Schrödinger 1925-1926). We have discussed before [2], by means of our definition of Feynman path integrals [3] the way in which the solutions of the quantum equations of motion are connected to classical motions in the precise sense of asymptotic expansion in powers of Planck's constant.

In the present paper we are concerned with the relation of the eigenvalue

* Partially supported by the USP-Mathematisierung, University of Bielefeld (Forschungsprojekt "Unendlich dimensionale Analysis") 
spectrum of the quantum mechanical energy operator with the orbits of the corresponding classical system. The simplest relations of this type were at the very basis of the "quantization rules" of Bohr and Sommerfeld, see e.g. [31]. For history and extensive references to other work on topics connected with the present paper see [5]. We shall discuss the general problem in the present paper using our method of stationary phase for oscillatory integrals in infinitely many dimensions [1], a mathematical version thus of the original ideas associated with Feynman path integrals. We get asymptotic expansions in powers of $h$ for the theta function $\theta(t)=\sum_{n} \exp \left(-\frac{i}{h} t \lambda_{n}\right)$ associated with the Schrödinger operator $H=-\frac{h^{2}}{2} \Delta$ $+\frac{1}{2}\left(x, A^{2} x\right)_{d}+V(x), A^{2}$ being a symmetric strictly positive matrix in $\mathbb{R}^{d}, x \in \mathbb{R}^{d}, \Delta$ the Laplacian in $\mathbb{R}^{d}, V(x)$ a bounded continuous potential on $\mathbb{R}^{d}, \lambda_{n}$ being the eigenvalues of $H$. The expansion is obtained by expressing $\theta(t)$ in terms of oscillatory integrals over a Hilbert space of paths and using then our method of stationary phase for such integrals. We obtain in this way an asymptotic expansion in powers of $h$ in terms of the periodic orbits of the corresponding classical mechanical anharmonic oscillator. This representation is a natural extension of the Poisson formula for the classical theta function. From it we deduce a trace formula for other associated functions, e.g. the $\zeta$-function $\zeta(s)=\sum \frac{1}{\lambda_{n}^{s}}$.

Let us now describe shortly the structure of the paper. In Sect. 2 we give a representation of the Green's function for the time dependent Schrödinger equation in terms of the oscillatory integrals on infinite dimensional spaces studied in [1]. In Sect. 3 we derive from this the trace formula for the theta function $\theta(t)$ associated with the Schrödinger operator $H$. We show that $\theta(t)$ is a generalized function expressed by an oscillatory integral over a Hilbert space of closed paths, starting at time 0 and ending at time $t$ at the same place. In Sect. 4 we derive an asymptotic expression for small $h$ for the $\theta$-function, by using the trace formula and the theory of asymptotic expansion of oscillatory integrals we developed in [1]. In fact we get that $\theta(t)$ is given asymptotically for small $h$ as a sum over contributions coming from closed orbits of the corresponding classical system.

In Sect. 5 we give corresponding asymptotic formulae for small $h$ in terms of classical closed orbits for different quantities associated with the quantum mechanical Schrödinger operator. In particular we give formulae for $g\left(\frac{1}{h} \lambda_{h}^{h}\right)$, where $g$ has support around $\frac{1}{h} \lambda_{n}^{h}, \lambda_{n}^{h}$ being the $n^{\text {th }}$ eigenvalue of $H$ and we obtain the proof of the Bohr-Sommerfeld quantization formula. The above formulae are of the type of the Poisson formula for the theta function associated with the torus. We also define the $\zeta$-function associated with the Schrödinger operator and we get an asymptotic expression for it for small $h$ in terms of classical closed orbits. These results give in particular a natural extension of those obtained in the literature for compact manifolds (see e.g. $[9,13,14,17,18,20,21,24,28,30]$ ) to the case of Schrödinger operators in $\mathbb{R}^{d}$. Our results have been announced in [6], [7]. 
Note added. After completion of this work we received a preprint by Chazarain [12] which contains related results obtained by completely different methods.

\section{The Green's Function Expressed by Normalized Integrals}

Let us first recall the definition of a normalized integral with respect to a given bounded quadratic form, a particular case of the ones defined in [3] (Sect. 4, esp. 59-64) and [1]. Let $\mathscr{H}$ be a real separable Hilbert space and let $B$ be an everywhere defined bounded symmetric operator on $\mathscr{H}$. Assume $B^{-1}$ is also a bounded symmetric operator on $\mathscr{H}$. Let $\mathscr{F}(\mathscr{H})$ be the space of functions $f$ on $H$ which are Fourier transforms of complex measures on $\mathscr{H}$, i.e.

$$
f(\gamma)=\int_{\mathscr{H}} e^{i(\gamma, \alpha)} d \mu_{f}(\alpha)
$$

with (,) the scalar product in $\mathscr{H}$ and $\mu_{f}$ a bounded complex measure on $\mathscr{H}$, with finite total variation $\left\|\mu_{f}\right\| \equiv \int_{\mathscr{H}} d\left|\mu_{f}\right|(\alpha)$. The mapping $f \rightarrow \mu_{f}$ is one-to-one and continuous, $\mathfrak{F}(\mathscr{H})$ being given the norm $\|f\|_{0}=\left\|\mu_{f}\right\| \mathfrak{F}(\mathscr{H})$ is a Banach function algebra, whose elements are going to be the integrable functions with respect to the following integral $I(f)$. For $f \in \mathfrak{F}(\mathscr{H})$ define

$$
I(f) \equiv \int_{\mathscr{H}} \exp \left(\frac{i}{2}(\gamma, B \gamma)\right) f(\gamma) d \gamma \equiv \int_{\mathscr{H}} \exp \left(-\frac{i}{2}\left(\alpha, B^{-1} \alpha\right)\right) d \mu_{f}(\alpha) .
$$

The symbol on the left hand side is defined by the right hand side, which exists as an integral on $\mathscr{H}$. The complex-valued functional $f \rightarrow I(f)$ is shown to be linear, continuous, normalized $(I(1)=1)$ on the Banach algebra $\mathfrak{F}(\mathscr{H})$, in fact $\left|I\left(\prod_{j=1}^{n} f_{j}\right)\right|$ $\leqq \prod_{j=1}^{n}\left\|f_{j}\right\|_{0}$, and is an extension to the infinite dimensional case of

$$
\begin{aligned}
\int_{\mathbb{R}^{d}} \exp \left(\frac{i}{2}(\gamma, B \gamma)\right) f(\gamma) d \gamma= & (2 \pi)^{-d / 2}|\operatorname{Det} B|^{1 / 2} \\
& \cdot \exp \left(-i \frac{\pi}{4} \operatorname{sign} B\right) \int_{\mathbb{R}^{d}} \exp \left(\frac{i}{2}(\gamma, B \gamma)\right) f(\gamma) d \gamma
\end{aligned}
$$

where $\mid$ Det $B \mid$ is the absolute value of the determinant of the matrix $B$ in $\mathbb{R}^{d}$ and $\operatorname{sign} B$ is the signature of the matrix $B$ (i.e. the difference of the number of positive and negative eigenvalues of $B$ ). We also recall the behaviour under translations of the normalized integral expressed by

$$
\int_{\mathscr{H}} \exp \left(\frac{i}{2}(\gamma+\alpha, B(\gamma+\alpha))\right) f(\gamma+\alpha) d \gamma=\int_{\mathscr{H}} \exp \left(\frac{i}{2}(\gamma, B \gamma)\right) f(\gamma) d \gamma .
$$

For other properties of the normalized integrals $I(f)$, in particular a Fubini theorem about iterated integrations, see [3], [4], [1].

We shall now use this normalized integral to express quantities associated with anharmonic oscillators on $\mathbb{R}^{d}$. Consider the system (anharmonic oscillator on $\mathbb{R}^{d}$ ) 
given by the classical action

$$
S_{t}(\gamma)=\frac{1}{2} \int_{0}^{t} \dot{\gamma}^{2}(\tau) d \tau-\frac{1}{2} \int_{0}^{t} \gamma(\tau) \cdot A^{2} \gamma(\tau) d \tau-\int_{0}^{t} V(\gamma(\tau)) d \tau
$$

where $A^{2}$ is a strictly positive symmetric operator on $\mathbb{R}^{d}$ and $\gamma_{1} \cdot \gamma_{2}=\left(\gamma_{1}, \gamma_{2}\right)_{d}$ is the scalar product in $\mathbb{R}^{d}$, and assume that $V \in \mathfrak{F}\left(\mathbb{R}^{d}\right)$ and real. Consider the operator

$$
H=-\frac{h^{2}}{2} \Delta+\frac{1}{2} x \cdot A^{2} x+V(x)
$$

in $L^{2}\left(\mathbb{R}^{d}\right)$, with $x \in \mathbb{R}^{d}$, where $h$ is Planck's constant divided by $2 \pi$. $H$ is essentially self-adjoint on the domain $C_{0}^{\infty}\left(\mathbb{R}^{d}\right)$ and its spectrum $\sigma(H)$ is contained in [inf $V$, $+\infty)$ and, by a theorem of Friedrichs, it is discrete. Let $\varphi \in \mathfrak{F}\left(\mathbb{R}^{d}\right) \cap L^{2}\left(\mathbb{R}^{d}\right)$ and consider $\exp (-i t H / h)$. For $\varphi \in D(H)$ we have that $\Psi(t, x)=\exp (-i t H / h) \varphi(x)$ solves the Schrödinger equation

$$
\operatorname{ih} \frac{\partial}{\partial t} \Psi(t, x)=H \Psi(t, x)
$$

with initial condition $\Psi(0, x)=\varphi(x)$.

It was proved in [1] Sect. 5 Theorem 5.1 that for all values of $t$ such that $\cos A t$ is nonsingular one has

with

$$
\exp \left(-\frac{i}{h} t H\right) \varphi(x)=\Psi(t, x)=|\cos A t|^{-1 / 2} \int_{\mathscr{H}_{0}} \exp \left(\frac{i}{2}(\gamma, B \gamma)\right) f(\gamma) d \gamma
$$

$$
f(\gamma)=\exp \left(\frac{i}{2}(\beta, B \beta)\right) \exp \left(-\frac{i}{h} \int_{0}^{t} V(\gamma(\tau)+\beta(\tau)) d \tau\right) \varphi(\gamma(0)+\beta(0))
$$

and

$$
|\cos A t|^{-1 / 2}=|\operatorname{Det} \cos A t|^{-1 / 2} \exp \left(i \frac{\pi}{4} \operatorname{sign}(\cos A t)\right) .
$$

Here $\mathscr{H}_{0}$ is the real separable Hilbert space of absolutely continuous functions $\gamma$ from $[0, t]$ into $\mathbb{R}^{d}$, such that $\gamma(t)=0$, with finite kinetic energy $\frac{1}{2} \int_{0}^{t} \dot{\gamma}^{2}(\tau) d \tau$ and norm given by $|\gamma|^{2}=\int_{0}^{t} \dot{\gamma}^{2}(\tau) d \tau . B$ is the bounded symmetric operator defined everywhere on $\mathscr{H}_{0}$ given by

$$
(\gamma, B \gamma)=\frac{1}{h} \int_{0}^{t}\left[\dot{\gamma}^{2}(\tau)-\gamma(\tau) A^{2} \gamma(\tau)\right] d \tau .
$$

In this case $B^{-1}$ is bounded symmetric on $\mathscr{H}_{0} . \beta(\tau)$ is the path such that $\ddot{\beta}+A^{2} \beta=0$, $\beta(t)=x, \dot{\beta}(0)=0, \quad$ i.e. $\quad \beta(\tau) \equiv \frac{\cos A \tau}{\cos A t} x$. We observe $\quad(\beta, B \beta)=-\frac{1}{h} x \cdot \operatorname{tg} A t A x$. 
Thus we see that $\exp \left(-\frac{i}{h} t H\right) \varphi$ is expressed through an oscillatory integral of the type we considered in [1]. In particular we can apply to it the expansion theory developed in Sect. 4 of [1]. We remark that (2.3) can be written in an obviously symbolic notation

$$
\left(\exp \left(-\frac{i}{h} t H\right) \varphi\right)(x)=|\cos A t|^{-1 / 2} \int_{\gamma(t)=x} \exp \left(\frac{i}{h} S_{t}(\gamma)\right) \varphi(\gamma(0)) d \gamma .
$$

In this paper we are basically interested in discussing the relation between the spectrum $\sigma(H)$ of $H$ and the classical paths, i.e. solutions of the equations of motion of the classical system with classical aption (2.1). To do this it is convenient to consider the Green's function $K(t, x, y)$ for the Cauchy problem. We have

$$
\left(\exp \left(-\frac{i}{h} t H\right) \varphi\right)(x)=\int_{\mathbb{R}^{d}} K(t, x, y) \varphi(y) d y .
$$

We shall now express $K(t, x, y)$ as a normalized integral. We have the following

Theorem 2.1. The Green's function $K(t, x, y)$ for the Schrödinger equation

$$
\operatorname{ih} \frac{\partial}{\partial t} \Psi(t, x)=-\frac{h^{2}}{2} \Delta \Psi(t, x)+\frac{1}{2}\left(x, A^{2} x\right)_{d} \Psi(t, x)+V(x) \Psi(t, x)
$$

with $V \in \mathbb{F}\left(\mathbb{R}^{d}\right)$ and $A^{2}$ a symmetric strictly positive matrix in $\mathbb{B}^{d}$ is given by

$$
\begin{aligned}
K(t, x, y)= & K_{0}(t, x, y) \int_{\mathscr{H}_{0,0}} \exp \left(\frac{i}{2}(\gamma, B \gamma)\right) \\
& \cdot \exp \left(-\frac{i}{h} \int_{0}^{t} V(\gamma(\tau)+\alpha(x, y)(\tau)+\beta(\tau)) d \tau\right) d \gamma
\end{aligned}
$$

where

$$
\begin{aligned}
K_{0}(t, x, y)= & \left|\operatorname{Det} \frac{A}{\sin A t}\right|^{1 / 2}(2 \pi / h)^{-d / 2} \exp \left(i \frac{\pi}{4} \sigma(\sin A t)\right) \exp \left(\frac{i}{2 h}(x, A \operatorname{cotg} A t x)_{d}\right) \\
& \cdot \exp \left(-\frac{i}{h}\left(x, \frac{A}{\sin A t} y\right)_{d}\right) \exp \left(\frac{i}{2 h}(y, A \operatorname{cotg} A t y)_{d}\right)
\end{aligned}
$$

is the corresponding Green's function for the harmonic oscillator and

$$
\beta(\tau) \equiv \frac{\cos A \tau}{\cos A t} x \quad \text { and } \alpha(x, y) \equiv \eta_{(y-x / \cos A t)}
$$

with $\eta_{z}(\tau) \equiv[\cos A \tau-(\operatorname{cotg} A t \sin A \tau)] z$.

Proof of Theorem 2.1. We first observe that we have the direct splitting $\mathscr{H}_{0}=$ $\mathscr{H}_{0,0}+\mathscr{H}_{d}$, where direct is with respect to the quadratic form $(\gamma, B \gamma)$, i.e. any $\gamma \in \mathscr{H}_{0}$ can be written in the form $\gamma=\tilde{\gamma}+\eta_{y}$ with $\tilde{\gamma} \in \mathscr{H}_{0,0}$ and $\left(\eta_{y}, B \tilde{\gamma}\right)=0, \eta_{y} \in \mathscr{H}_{d}$. Here $\eta_{y}$ is the path

$$
\eta_{y}=\cos A \tau y-(\operatorname{cotg} A t \sin A \tau) y
$$


Equivalently $\eta_{y}=B^{-1} \gamma_{y}$, with $\gamma_{y} \in \mathscr{H}_{0,0}$, where $\mathscr{H}_{0,0}$ is the orthogonal complement of $\mathscr{H}_{0,0}$ in $\mathscr{H}_{0}$ with respect to the scalar product in $\mathscr{H}_{0}$. In this situation we can use the Fubini theorem about iteration of normalized integrals (Prop. 4.5 in [3]) and we get

$$
\begin{aligned}
\int_{\mathscr{H}_{0}} \exp \left(\frac{i}{2}(\gamma, B \gamma)\right) f(\gamma) d \gamma= & \int_{\mathscr{H}_{0}, 0 \oplus \mathscr{H}_{d}} \exp \left(\frac{i}{2}\left(\tilde{\gamma}+\eta_{y}, B\left(\tilde{\gamma}+\eta_{y}\right)\right)\right) f\left(\tilde{\gamma}+\eta_{y}\right) d \tilde{\gamma} d \eta_{y} \\
= & \int_{\mathscr{W}^{d}} \exp \left(\frac{i}{2 h}(y, A \operatorname{cotg} A t y)_{d}\right) \\
& \left.\cdot \int_{\mathscr{H}_{0,0}} \exp \left(\frac{i}{2}(\tilde{\gamma}, B \tilde{\gamma})\right) f\left(\tilde{\gamma}+\eta_{y}\right) d \tilde{\gamma}\right) d y
\end{aligned}
$$

where we observed that $\mathscr{H}_{d} \cong \mathbb{R}^{d}$ by the natural mapping $\eta_{y} \leftrightarrow y$.

Introducing now (2.4) for $f$ we can rewrite the right hand side of (2.7) in the form

$$
\begin{gathered}
\exp \left(-\frac{i}{2 h}(x, \operatorname{tg} A t A x)_{d}\right) \int_{\mathbb{B}^{d}} \exp \left(\frac{i}{2 h}(y, A \operatorname{cotg} A t y)_{d}\right) \int_{\mathscr{H}_{0,0}} \exp \left(\frac{i}{2}(\tilde{\gamma}, B \tilde{\gamma})\right) \\
\left.\cdot \exp \left(-\frac{i}{h} \int_{0}^{t} V\left(\tilde{\gamma}(\tau)+\eta_{y}(\tau)+\beta(\tau)\right) d \tau\right)\left(\varphi(\tilde{\gamma})(0)+\eta_{y}(0)+\beta(0)\right) d \tilde{\gamma}\right) d y .
\end{gathered}
$$

We now observe that we have

$$
\tilde{\gamma}(0)+\eta_{y}(0)+\beta(0)=y+\frac{x}{\cos A t},
$$

and setting $z=y+\frac{x}{\cos A t}$ we can rewrite (2.8) in the form

$$
\begin{aligned}
\exp \left(-\frac{i}{2 h}(x, \operatorname{tg} A t A x)_{d}\right) \exp \left(-\frac{i}{h}\left(x, \frac{A}{\sin A t} z\right)_{d}\right) \exp \left(\frac{i}{2 h}\left(x, \frac{A}{\cos A t \sin A t} x\right)_{d}\right) \\
\cdot \int_{\mathbb{R}^{d}} \exp \left(\frac{i}{2 h}(z, A \operatorname{cotg} A t z)_{d}\right)\left(\int_{\mathscr{H}_{0,0}} \exp \left(\frac{i}{2}(\tilde{\gamma}, B \tilde{\gamma})\right)\right. \\
\left.\quad \cdot \exp \left(-\frac{i}{h} \int_{0}^{t} V\left(\tilde{\gamma}(\tau)+\eta_{(z-x / \cos A t)}(\tau)+\beta(\tau)\right) d \tau\right) \varphi(z) d \tilde{\gamma}\right) d z
\end{aligned}
$$

Hence we see that (2.7) multiplied by $|\cos A t|^{-1 / 2}$, i.e. (2.3), is equal to

with

$$
\int_{\mathbb{R}^{d}} K(t, x, z) \varphi(z) d z
$$

$$
\begin{aligned}
K(t, x, z)= & K_{0}(t, x, z) \int_{\mathscr{H}_{0,0}} \exp \left(\frac{i}{2}(\tilde{\gamma}, B \tilde{\gamma})\right) \\
& \cdot \exp \left(-\frac{i}{h} \int_{0}^{t} V\left(\tilde{\gamma}(\tau)+\eta_{(z-x / \cos A t)}(\tau)+\beta(\tau)\right) d \tau\right) d \tilde{\gamma}
\end{aligned}
$$


where

$$
\begin{aligned}
K_{0}(t, x, z) \equiv & \exp \left(\frac{i}{2 h}(x, A \operatorname{cotg} A t x)_{d}\right) \exp \left(-\frac{i}{h}\left(x, \frac{A}{\sin A t} z\right)_{d}\right) \\
& \cdot \exp \left(\frac{i}{2 h}(z, A \operatorname{cotg} A t z)_{d}\right)\left|\operatorname{Det} \frac{A}{\sin A t}\right|^{1 / 2}(2 \pi)^{-d / 2} \\
& \cdot \exp \left(i \frac{\pi}{4} \sigma(\sin A t)\right) .
\end{aligned}
$$

The factor $\left|\operatorname{Det} \frac{A}{\sin A t}\right|^{1 / 2}(2 \pi)^{-d / 2} \exp \left(i \frac{\pi}{4} \sigma(\sin A t)\right)$ is actually due to the fact that the normalized integral over $\mathbb{R}^{d}$ has been expressed in (2.11) by a Lebesgue integral over $\mathbb{R}^{d}$, by

$$
\int_{\mathbb{R}^{d}}=\frac{|\operatorname{det}(A \operatorname{cotg} A t)|^{1 / 2}}{(2 \pi)^{d / 2}} \exp \left(i \frac{\pi}{4} \sigma(\sin A t)\right) \int_{\mathbb{R}^{d}} .
$$

Note that $K_{0}$ is the Green's function for the Schrödinger equation (2.2) with $V=0$, i.e. for the harmonic oscillator in $d$-dimensions. From (2.3), (2.4), (2.7)-(2.13), we see that $K(t, x, z)$ is the kernel of the unitary operator $\exp \left(-\frac{i}{h} t H\right)$ in $L^{2}\left(\mathbb{R}^{d}, d x\right)$. This proves the theorem.

Remark. In terms of the symbolic notation used in (2.5) we can write the formula for the Green's function in Theorem 2.1 in the form

where

$$
K(t, x, y)=\int_{\substack{\gamma(t)=x \\ \gamma(0)=y}} \exp \left(\frac{i}{h} S_{t}(\gamma)\right) d \gamma
$$

$$
S_{t}(\gamma)=\frac{1}{2} \int_{0}^{t} \dot{\gamma}^{2}(\tau) d \tau-\frac{1}{2} \int_{0}^{t}\left(\gamma^{\circ} A^{2} \gamma\right)_{d} d \tau-\int_{0}^{t} V(\gamma(\tau)) d \tau
$$

\section{The Trace Formula}

As remarked in the preceding section the spectrum of $H$ as given by (2.2) is discrete. Let us call $\lambda_{n}, n=0,1,2, \ldots$, the eigenvalues of $H$. Here we take $h=1$. We have $\lambda_{0}=\inf \sigma(H) \geqq-\frac{1}{2} \operatorname{tr} A-\inf V(x)$ and $\lambda_{0} \leqq \lambda_{1} \leqq \lambda_{2} \leqq \ldots, \lambda_{n} \uparrow \infty$. We will now give a meaning to the formal expression

$$
\operatorname{Tr} e^{-i t H}=\sum_{n=0}^{\infty} e^{-t t \lambda_{n}}
$$

Although $e^{-i t H}$ is not of trace class we shall see that these expressions are well defined in the sense of generalized functions, and are expressed by oscillatory integrals.

We first observe that the distributional kernel of $e^{-i t H}$ is given by

$$
e^{-i t H}(x, y)=K(t, x, y)=\sum_{k=0}^{\infty} e^{-i t \lambda_{k}} \bar{f}_{k}(x) f_{k}(y),
$$


where $\left\{f_{k}\right\}$ is a basis of normalized eigenfunctions in $L^{2}\left(\mathbb{R}^{d}, d x\right), f_{k}$ being the eigenfunction of $H$ corresponding to the eigenvalue $\lambda_{k}$. On the other hand we have seen in Theorem 2.1 that $K(t, x, y)$ is expressible by normalized integrals. Introducing this, which we write for shortness as in (2.14), into (3.1), we get the distributional equality

$$
e^{-i t H}(x, y)=\sum_{k} \bar{f}_{k}(x) e^{-i t \lambda_{k}} f_{k}(y)=\int_{\substack{\gamma(t)=x \\ \gamma(0)=y}} e^{i S_{\mathrm{t}}(\gamma)} d \gamma .
$$

On the other hand

$$
\left(f_{k}, e^{-i t H} f_{k}\right)=e^{-i t \lambda_{k}},
$$

since the $f_{k}$ are normalized eigenfunctions of $H$, where (,) is the scalar product in $L^{2}\left(\mathbb{R}^{d}, d x\right)$. But by the definition of $e^{-i t H}(x, y)$, the left hand side of (3.3) is equal to

$$
\int_{\mathbb{R}^{d}} \bar{f}_{k}(x)\left(\int_{\mathbb{R}^{d}} e^{-i t H}(x, y) f_{k}(y) d y\right) d x=\left\langle e^{-i t H}, \bar{f}_{k} \otimes f_{k}\right\rangle .
$$

Integrating the bounded continuous function (3.3) against an integrable $\varphi$ with Fourier transform $\tilde{\varphi}$ continuous with compact support we have, using (3.4)

$$
\begin{aligned}
\tilde{\varphi}\left(\lambda_{k}\right) & =\int_{\mathbb{R}} \varphi(t) e^{-i t \lambda_{k}} d t=\int_{\mathbb{R}} \varphi(t)\left(\int_{\mathbb{R}^{d}} \bar{f}_{k}(x) \int_{\mathbb{R}^{d}} e^{-i t I}(x, y) f_{k}(y) d y\right) d t \\
& =\int_{\mathbb{R}} \varphi(\mathrm{t})\left\langle\mathrm{e}^{i t H}, \bar{f}_{k} \otimes f_{k}\right\rangle d t,
\end{aligned}
$$

hence

$$
\sum_{k} \tilde{\varphi}\left(\hat{\lambda}_{k}\right)=\int_{\mathbb{R}} \varphi(t)\left\langle e^{-i t H}, \sum_{k} \bar{f}_{k} \otimes f_{k}\right\rangle d t
$$

Using the completeness of the $\left\{f_{k}\right\}$ we get then

$$
\sum_{k} \tilde{\varphi}\left(\lambda_{k}\right)=\int_{\mathbb{B}} \varphi(t)\left(\int_{\mathbb{R}^{d}} e^{-t t H}(x, x) d x\right) d t .
$$

Note that the left hand side is finite by the assumptions on $\varphi$, hence $\int_{\mathbb{R}^{d}} e^{-i t H}(x, x) d x$ is a well defined generalized function of $t$. On the other hand from (3.2) we see that this distribution can be written also in the form

$$
\int_{\substack{\gamma(t)=x \\ \gamma(0)=x}} e^{i S_{\mathrm{t}}(\gamma)} d \gamma
$$

where (3.8) is by (2.14) and Theorem 2.1 expressible by a normalized integral over the Hilbert space $\mathscr{H}_{0,0}$ of closed paths.

From (3.7), (3.8) and Theorem 2.1 and the fact that $\tilde{\varphi}$ is the Fourier transform of $\varphi$, we have thus the equality in the sense of generalized functions in $t$

$$
\sum_{k} e^{-i t \lambda_{k}}=\int_{\mathbb{R}^{d}} e^{-i t H}(x, x) d x=\int_{\mathbb{R}^{d}}\left(\int_{\substack{\gamma(t)=x \\ \gamma(0)=x}} e^{i S_{t}(\gamma)} d \gamma\right) d x
$$




$$
=\int_{\mathbb{R}^{d}} K_{0}(t, x, x)\left[\int_{\mathscr{H}_{0,0}} \exp \left(\frac{i}{2}(\gamma, B \gamma)\right) g_{x}(\gamma) d \gamma\right] d x,
$$

where $g_{x}(\gamma)=\exp \left(-i \int_{0}^{t}[V(\gamma(\tau)+\alpha(x)(\tau)+\beta(\tau)] d \tau)\right.$ and $\alpha(x)(\tau) \equiv \alpha(x, x)(\tau)$. This will now be taken as the definition of $\operatorname{Tr} e^{-t t H} \equiv \theta(t)$ and we shall call $\theta(t)$ the theta function for the Schrödinger operator $H$.

Remark 1. In the integral (3.9) the normalized integral over $\mathscr{H}_{0,0}$ exists as a continuous function of $t$ and $x$ and it is only after integration over $x$ that the formula has to be interpreted in the generalized sense.

Let us formulate the above result as

Theorem 3.1. Let $H=-\frac{1}{2} \Delta+\frac{1}{2}\left(x, A^{2} x\right)_{d}+V(x)$, with $A^{2}$ a symmetric strictly positive $d \times d$ matrix and $V \in \mathbb{F}\left(\mathbb{R}^{d}\right)$. Let $\lambda_{k}$ be the eigenvalues of $H$. Then in the sense of generalized functions in $t$ we have

$$
\theta(t) \equiv \operatorname{Tr} e^{-i t H} \equiv \sum_{k} e^{-i t \lambda_{k}}=\int_{\mathbb{\mathbb { B } ^ { d }}}\left(\int_{\substack{\gamma(t)=x \\ \gamma(0)=x}}^{\tilde{j}} e^{i S_{\mathrm{t}}(\gamma)} d \gamma\right) d x,
$$

where the path integral is defined by

$$
\int_{\substack{\gamma(t)=x \\ \gamma(0)=x}} e^{i S_{t}(\gamma)} d \gamma \equiv K_{0}(t, x, x) \int_{\mathscr{H}_{0,0}} \exp \left(\frac{i}{2}(\gamma, B \gamma)\right) g_{x}(\gamma) d \gamma,
$$

where $S_{t}(\gamma)$ is the action along $\gamma, \mathscr{H}_{0,0}$ is the Hilbert space of paths of finite kinetic energy beginning and ending at the origin,

$$
\begin{aligned}
K_{0}(t, x, x)= & \left|\operatorname{det} \frac{A}{\sin A t}\right|^{1 / 2}\left((2 \pi)^{d / 2}\right)^{-1} \exp \left(i\left(x, \frac{A}{\sin A t}(\cos A t-1) x\right)_{d}\right) \\
& \cdot \exp \left(i \frac{\pi}{4} \sigma(\sin A t)\right), \\
& g_{x}(\gamma)=\exp \left[i \int_{0}^{t} V(\gamma(\tau)+\alpha(x)(\tau)+\beta(\tau)) d \tau\right],
\end{aligned}
$$

where

$$
\alpha(x)(\tau) \equiv[\cos A \tau-\operatorname{cotg} A t \sin A \tau]\left(x-\frac{x}{\cos A t}\right) .
$$

Remark. In the case where $H$ is the Laplacian on a torus $\mathbb{R}^{d} / \Gamma$, then $\theta(t)$ is the analytic continuation to $\tau=$ it of the classical theta function $\theta(\tau)=\sum_{m \in \mathbb{Z}^{n}} e^{-\tau m^{2}}$

\section{The Asymptotic Expansions in Terms of Periodic Orbits}

We shall now derive asymptotic expressions for small $h$ of the quantities entering the trace formula of the preceding sections, where $h$ is the parameter (in the physical 
world Planck's constant divided by $2 \pi$ ) which appears in Schrödinger's equation as follows

$$
\operatorname{ih} \frac{\partial}{\partial t} \Psi(t, x)=\left[-\frac{h^{2}}{2} \Delta+\frac{1}{2}\left(x, A^{2} x\right)_{d}+V(x)\right] \Psi(t, x),
$$

where $(,)_{d}$ is the scalar product in $\mathbb{R}^{d}$.

We start by considering the trace formula given by Theorem 3.1, which in the present case reads

$$
\operatorname{Tr} \exp \left(-\frac{i}{h} t H\right)=\int_{\mathbb{R}^{d}}\left(\int_{\substack{\gamma(t)=x \\ \gamma(0)=x}} \exp \left(\frac{i}{h} S_{t}(\gamma)\right) d \gamma\right) d x
$$

the path integral being defined by

$$
\int_{\substack{\gamma(t)=x \\ \gamma(0)=x}} \exp \left(\frac{i}{h} S_{t}(\gamma)\right) d \gamma=K^{h}(t, x, x)=K_{0}^{h}(t, x, x) \int_{\mathscr{H}_{0,0}} \exp \left(\frac{i}{2}(\gamma, B \gamma)\right) g_{x}^{h}(\gamma) d \gamma,
$$

where

$$
\begin{aligned}
K_{0}^{h}(t, x, x) \equiv & \frac{1}{(2 \pi h)^{d / 2}}\left|\operatorname{det} \frac{A}{\sin A t}\right|^{1 / 2} \exp \left(\frac{i}{h}\left(x, \frac{A}{\sin A t}(\cos A t-1) x\right)_{d}\right) \\
& \cdot \exp \left(i \frac{\pi}{4} \sigma(\sin A t)\right), \\
& g_{x}^{h}(\gamma) \equiv \exp \left(-\frac{i}{h} \int_{0}^{t} V(\gamma(\tau)+\alpha(x)(\tau)+\beta(\tau)) d \tau\right),
\end{aligned}
$$

and we recall that

$$
(\gamma, B \gamma) \equiv \frac{1}{\mathrm{~h}} \int_{0}^{t}\left[\dot{\gamma}^{2}(\tau)-\left(\gamma(\tau), A^{2} \gamma(\tau)\right)_{d}\right] d \tau
$$

Note that now $\mathscr{H}_{0,0}$ is equipped with the scalar product given by the norm $\frac{1}{h} \int_{0}^{t} \dot{\gamma}^{2}(\tau) d \tau$. The operator $B$ is clearly bounded and is invertible with bounded inverse provided the equation $\ddot{\gamma}+A^{2} \gamma=0, \gamma(t)=\gamma(0)=0$ has no nontrivial solution, which is the case if $t$ is not a multiple of the periods $\pi / \lambda_{i}$ of the corresponding classical harmonic oscillator, where the $\lambda_{i}$ are the eigenvalues of $A$. In this case the integral over $\mathscr{H}_{0,0}$ is of the type of those considered in Sect. 4 of [1], since $B^{-1}$ is bounded. We now proceed as in Sect. 4 of [1] and will prove that the integral (4.3) can be evaluated asymptotically for $h \rightarrow 0$, reducing the situation to the one of an oscillatory integral with one stationary point followed by a finite dimensional one.

We now take an integer $n$ and split any path $\gamma \in \mathscr{H}_{0,0}$ into $\gamma=\gamma_{1}+\gamma_{2}$, where $\gamma_{i} \in \mathscr{H}_{0,0}, i=1,2, \gamma_{2}$ is such that $\ddot{\gamma}_{2}(\tau)+A^{2} \gamma_{2}(\tau)=0$ in all intervals $\left(\frac{k-1}{n} t, k \frac{t}{n}\right)$ $k=1,2, \ldots, n$ with $\gamma_{2}\left(k \frac{t}{n}\right)=\gamma\left(k \frac{t}{n}\right)$ for all $k=0, \ldots, n$. Note that then $\gamma_{2}(0)=$ 
$\gamma_{2}(t)=0$, and $\gamma_{1} \equiv \gamma-\gamma_{2}$, hence $\gamma_{1}(k t)=0, k=0, \ldots, n$. Hence the space of all $\gamma_{2}$ when $\gamma$ varies in $\mathscr{H}_{0,0}$ is $(n-1) d$-dimensional. Moreover note that $\left(\gamma_{2}, B \gamma_{1}\right)=0$, since on each interval $\left[(k-1) \frac{t}{n}, k \frac{t}{n}\right] k=1, \ldots, n, \gamma_{2}$ is a solution of $\ddot{\gamma}+A^{2} \gamma=0$ in the inner points and the limit of its derivative at the endpoints exist and $\gamma_{1}$ vanish at both endpoints. Hence $\gamma=\gamma_{1}+\gamma_{2}$ is a direct splitting of $\mathscr{H}_{0,0}$ with respect to the quadratic form $(\gamma, B \gamma)$, which together with the fact that the quadratic form $B$ is nondegenerate on $\mathscr{H}_{1} \equiv B^{-1} \mathscr{H}_{2}$ allows us to use by Proposition 4.5 in [3] the Fubini theorem for normalized integrals and split the integral over $\mathscr{H}_{0,0}$ in (4.3) into the iterated integral first over $\mathscr{H}_{1}$ and then over $\mathscr{H}_{2}$, where $\mathscr{H}_{2}$ is the $(n-1) d$-dimensional Hilbert space of the paths of above form $\gamma_{2}$ (and $\mathscr{H}_{1}$ is the Hilbert space of paths $\gamma_{1}$ of the above form).

We will now proceed to examine the integral over $\mathscr{H}_{0,0}$. We have

$$
\begin{aligned}
& \int_{\mathscr{H}_{0,0}} \exp \left(\frac{i}{2}(\gamma, B \gamma)\right) g_{x}^{h}(\gamma) d \gamma=\int_{\mathscr{H}_{2}} \exp \left(\frac{i}{2}\left(\gamma_{2}, B \gamma_{2}\right)\right)\left(\int_{\mathscr{H}_{1}} \exp \left(\frac{i}{2}\left(\gamma_{1}, B \gamma_{1}\right)\right)\right. \\
& \left.\cdot \exp \left(-\frac{i}{h} \int_{0}^{t} V\left(\gamma_{1}(\tau)+\gamma_{2}(\tau)+\alpha(x)(\tau)+\beta(\tau)\right) d \tau\right) d \gamma_{1}\right) d \gamma_{2}
\end{aligned}
$$

Using now that $V$ is the Fourier transform of the measure $\mu_{v}$ on $\mathbb{R}^{d}$ we have that (4.4) is equal to

$$
\int_{\mathscr{H}_{2}} \exp \left(\frac{i}{2}\left(\gamma_{2}, B \gamma_{2}\right)\right)\left(\int_{\mathscr{H}_{1}} \exp \left(\frac{i}{2}\left(\gamma_{1}, B \gamma_{1}\right)\right) \exp \left(-\frac{i}{h} W\left(\gamma_{1}, \gamma_{2}, x\right)\right) d \gamma_{1}\right) d \gamma_{2}
$$

with

$$
\left.W\left(\gamma_{1}, \gamma_{2}, x\right) \equiv \int_{0}^{t} \int_{\mathbb{R}^{d}} \exp i\left(\sigma, \gamma_{1}(\tau)+\gamma_{2}(\tau)+\alpha(x)(\tau)+\beta(\tau)\right)_{d}\right) d \mu_{V}(\sigma) d \tau .
$$

Introducing now the function $\gamma_{\tau}(s) \equiv G(s, \tau)$, with $G(s, \tau)$ the Green's function for $-\frac{d^{2}}{d \tau^{2}}$ with Dirichlet boundary conditions at $\tau=0$ and $\tau=t$, i.e. $G(s, \tau)=\frac{s(t-\tau)}{t}$ for $s \leqq \tau$, we have

$$
\left(\sigma, \gamma_{1}(\tau)+\gamma_{2}(\tau)\right)_{d}=\left(\sigma \gamma_{\tau}, \gamma_{1}+\gamma_{2}\right)
$$

where we recall that (,) denotes the scalar product in $\mathscr{H}_{0}$, hence in $\mathscr{H}_{0,0}$. From (4.7) we can express $W$ as the Fourier transform on $\mathscr{H}_{0,0}$ of the measure

$$
d \mu_{W}(\rho)=\int_{0}^{t} \int_{\mathbb{B}^{d}} \delta_{\sigma \gamma_{\tau}}(\rho) e^{i\left(\sigma \gamma_{\tau}, \alpha(x)+\beta\right)} d \mu_{V}(\sigma) d \tau,
$$

From (4.6) we can trivially estimate the total variation of $\mu_{W}$ on $\mathscr{H}_{0,0}$ by the total variation of $\mu_{V}$ on $\mathbb{R}^{d}$ by

$$
\left\|\mu_{W}\right\| \leqq t\left\|\mu_{V}\right\|
$$


To apply Theorem 4.3 of [1] we need moreover to show that the quantity

$$
\int_{\mathscr{H}_{0,0}} \exp \left(\sqrt{2\left\|B^{-1}\right\|} \lambda\left|\rho_{1}\right|\right) d\left|\mu_{W}\right|\left(\rho_{1}, \rho_{2}\right)
$$

where $\rho_{1}, \rho_{2}$ are the components of $\left(\rho_{1}, \rho_{2}\right) \in \mathscr{H}_{0,0}$ according to the splitting $\mathscr{H}_{0,0}=\mathscr{H}_{1} \oplus \mathscr{H}_{2}$ described above, is bounded by $\lambda^{2}$, for some $\lambda>0$ such that $\left\|\mu_{W}\right\|<\lambda^{2}$. We recall that $|\cdot|$ is the norm in $\mathscr{H}_{0}$, hence in $\mathscr{H}_{0,0}$ as well. For this bound it is sufficient that

$$
\int_{\mathscr{H}_{0,0}} \exp \left(\sqrt{2\left\|B^{-1}\right\|} \lambda\left|\rho_{1}\right|\right) d\left|\mu_{W}\right|\left(\rho_{1}, \rho_{2}\right)<\lambda^{2} .
$$

Now from (4.8) we have

$$
\begin{aligned}
d \mu_{w}\left(\rho_{1}, \rho_{2}\right)= & \int_{0}^{t} \int_{\mathbb{R}^{d}} \delta_{\sigma \gamma_{\tau}}\left(\rho_{1}, \rho_{2}\right) e^{i\left(\sigma \gamma_{\tau}, \alpha(x)\right)} e^{i\left(\sigma \gamma_{\tau}, \beta\right)} d \mu_{V}(\sigma) d \tau \\
= & \int_{0}^{t} \int_{\mathbb{R}^{d}} \delta_{\sigma \gamma_{t}^{(1)}}\left(\rho_{1}\right) e^{i\left(\sigma \gamma_{\tau}^{(1)}, \alpha(x)+\beta\right)} \\
& \cdot \delta_{\sigma \gamma_{t}^{(2)}}\left(\rho_{2}\right) e^{i\left(\sigma \gamma_{t}^{(2)}, \alpha(x)+\beta\right)} d \mu_{V}(\sigma) d \tau,
\end{aligned}
$$

where $\sigma \gamma_{\tau}^{(1)}, \sigma \gamma_{\tau}^{(2)}$ are the components of $\sigma \gamma_{\tau}$ in $\mathscr{H}_{1}$ respectively $\mathscr{H}_{2}$, according to the above decomposition.

But the measure $\exp \left(\sqrt{2\left\|B^{-1}\right\|} \lambda\left|\rho_{1}\right|\right) \mu_{W}\left(\rho_{1}, \rho_{2}\right)$ is equal to

$$
\begin{aligned}
\int_{0}^{t} \int_{\mathbb{R}^{d}} & \exp \left(\sqrt{2\left\|B^{-1}\right\|} \lambda\left|\sigma \gamma_{\tau}^{(1)}\right|\right) \delta_{\sigma \gamma_{t}^{(1)}}\left(\rho_{1}\right) e^{i\left(\sigma \gamma_{\mathrm{t}}^{(1)}, \alpha(x)+\beta\right)} \delta_{\sigma \gamma_{t}^{(2)}}\left(\rho_{2}\right) e^{\left.i\left(\sigma \gamma t^{2}\right), \alpha(x)+\beta\right)} \\
= & \sum_{k=1}^{n} \int_{(k-1)(t / n) \mathbb{R}^{d}}^{k(t / n)} \exp \left(\sqrt{2\left\|B^{-1}\right\|} \lambda\left|\sigma \gamma_{\tau}^{(1)}\right|\right) \delta_{\sigma \gamma_{t}^{(1)}}\left(\rho_{1}\right) \delta_{\sigma \gamma_{t}^{(2)}}\left(\rho_{2}\right) e^{i\left(\sigma \gamma_{t}^{(1)}, \alpha(x)+\beta\right)} \\
& \cdot e^{i\left(\sigma \gamma_{t}^{(2)}, \alpha(x)+\beta\right)} d \mu_{V}(\sigma) d \tau .
\end{aligned}
$$

But

$$
\left|\sigma \gamma_{\tau}^{(1)}\right|^{2}=\left(\sigma, \sigma \gamma_{\tau}^{(1)}\right)_{d}=\int_{k(t / n)}^{\tau} \dot{\gamma}_{\tau}^{(1)}(s) d s|\sigma|_{d}^{2} \leqq \int_{k(t / n)}^{\tau}\left|\dot{\gamma}_{\tau}^{(1)}(s)\right| d s|\sigma|_{d}^{2} \leqq \sqrt{(\tau-k(t / n))}\left|\sigma \gamma_{\tau}^{(1)}\right||\sigma|_{d}
$$

for all $\tau \in[(k-1)(t / n), k(t / n)] \quad k=0,1, \ldots, n$, where we used $\left(\sigma \gamma_{\tau}^{(1)}, \sigma \gamma_{\tau}^{(1)}\right)=$ $\left(\sigma, \sigma \gamma_{\tau}^{(1)}(\tau)\right)_{d}$. Now the total variation of (4.12) is bounded by

$$
\left.\sum_{k=1}^{n} \int_{(k-1)(t / n)}^{k(t / n)} \int_{\mathbb{R}^{d}} \exp \sqrt{2\left\|B^{-1}\right\|} \lambda\left|\sigma \gamma_{\tau}^{(1)}\right|\right) d\left|\mu_{V}\right|(\sigma) d \tau \text {. }
$$

From (4.13) we have then that (4.14) is bounded by

$$
\begin{aligned}
& \sum_{k=1}^{n} \frac{t}{n} \int_{\mathbb{R}^{d}} \sup _{\tau \in[(k-1)(t / n), k(t / n)]} \exp \left(\sqrt{2\left\|B^{-1}\right\|} \lambda|\sigma|_{d}^{1 / 2} \sqrt{\frac{t}{n}}\left|\sigma \gamma_{\tau}^{(1)}\right|^{1 / 2}\right) d\left|\mu_{V}\right|(\sigma) \\
& \leqq t \int_{\mathbb{R}^{d}} \exp \left(\sqrt{2\left\|B^{-1}\right\|} \lambda \sqrt{\frac{t}{n} \frac{t^{1 / 4}}{\sqrt{2}}|\sigma|_{d}}\right) d\left|\mu_{V}\right|(\sigma),
\end{aligned}
$$


where we used $\left|\sigma \gamma_{\tau}^{(1)}\right| \leqq\left|\sigma \gamma_{\tau}\right|=\gamma_{\tau}(\tau)^{1 / 2}|\sigma|_{d}=\left(\frac{\tau(t-\tau)}{t}\right)^{1 / 2}|\sigma|_{d} \leqq \frac{\sqrt{t}}{2}|\sigma|_{d}$. If we assume now that $V$ is such that

$$
\int_{\mathbb{R}^{d}} e^{\varepsilon|\sigma|_{d}} d\left|\mu_{V}\right|(\sigma)<+\infty
$$

for some $\varepsilon>0$, we see that for any fixed $t$ we can choose $n$ and $\lambda>0, n$ sufficiently big, such that (4.15) is bounded by $\lambda^{2}$. On the other hand by (4.9) we have $\left\|\mu_{W}\right\|<\lambda^{2}$, whenever $\lambda^{2}>t\left\|\mu_{V}\right\|$ (and $\left\|\mu_{V}\right\|$ is finite, independent of $\lambda$ by (4.16)).

We have thus verified all basic conditions required for the results in Sect. 4 of [1]. Those results give then the general asymptotic representation of the oscillatory integral over $\mathscr{H}_{0,0}$ as $h \rightarrow 0$, as a sum of terms which represent contributions from each stationary point of the phase

$$
\Phi(\gamma) \equiv \int_{0}^{t}\left\{\frac{1}{2} \dot{\gamma}^{2}(\tau)-\left(\gamma(\tau), A^{2} \gamma(\tau)\right)_{d}-V(\gamma(\tau)+\alpha(x)(\tau)+\beta(\tau))\right\} d \tau
$$

Note that this phase $\Phi(\gamma)$ depends on $x$, through the second term. Now the stationary points of the phase $\Phi(\gamma)$ are the solutions of $d \Phi(\gamma)=0$. This is equivalent with

$$
B \gamma=d W(\gamma+\alpha(x)+\beta), \quad \text { with } \quad W(\gamma+\alpha(x)+\beta) \equiv \int_{0}^{t} V(\gamma(\tau)+\alpha(x)(\tau)+\beta(\tau)) d \tau
$$

But (4.17) is equivalent with Newton's equàtion of motion

$$
\ddot{\gamma}_{x}^{t}(\tau)+A^{2} \gamma_{x}^{t}(\tau)=-\nabla V\left(\gamma_{x}^{t}(\tau)\right)
$$

for a particle in the total potential $\frac{1}{2}\left(z, A^{2} z\right)_{d}+V(z)$ and with boundary conditions $\gamma_{x}^{t}(0)=\gamma_{x}^{t}(t)=x$. We shall say that such a solution $\gamma_{x}^{t}$ of (4.18) is nondegenerate if $D\left(\gamma_{x}^{t}\right) \equiv 1-B^{-1} d^{2} W\left(\tilde{\gamma}_{x}^{t}\right)$ is nondegenerate, where $\tilde{\gamma}_{x}^{t}=\gamma_{x}^{t}-\alpha(x)-\beta$. We shall call a solution $\gamma_{x}^{t}$ a closed orbit if it has finite energy. We can now formulate the following

Lemma 4.1. Let $V \in \mathbb{F}\left(\mathbb{R}^{d}\right)$ be such that

$$
\int_{\mathbb{R}^{d}} e^{\varepsilon|\alpha|_{d}} d\left|\mu_{V}\right|(\alpha)<+\infty
$$

for some $\varepsilon>0$. Then we have for the diagonal part $K^{h}(t, x, x)$ of the Green's function for the Schrödinger equation the following asymptotic behaviour as $h \rightarrow 0$

$$
\begin{aligned}
K^{h}(t, x, x)= & \int_{\substack{\gamma(t)=x \\
\gamma(0)=x}} \exp \left(\frac{i}{h} S_{t}(\gamma)\right) d \gamma \\
= & \sum \frac{\exp \left(\frac{i}{h} S_{t}\left(\gamma_{x}^{t}\right)\right) \exp \left(-i \frac{\pi}{2} m\left(\gamma_{x}^{t}\right)\right)}{\left|D\left(\gamma_{x}^{t}\right)\right|^{1 / 2}(2 \pi i h)^{d / 2}} \\
& \cdot(1+h O(1)), \\
\left|D\left(\gamma_{x}^{t}\right)\right|^{1 / 2} & \left|\operatorname{det} \frac{A}{\sin A t}\right|^{-1 / 2}\left|\operatorname{Det}\left(1-B^{-1} d^{2} W\left(\tilde{\gamma}_{x}^{t}\right)\right)\right|,
\end{aligned}
$$


where the sum is over all closed orbits which solve the equation of motion

$$
\ddot{\gamma}_{x}^{t}(\tau)+A^{2} \gamma_{x}^{t}(\tau)=-\nabla V\left(\gamma_{x}^{t}(\tau)\right)
$$

with $\gamma_{x}^{t}(t)=\gamma_{x}^{t}(0)=x$ and $\tilde{\gamma}_{x}^{t} \equiv \gamma_{x}^{t}-\alpha(x)-\beta$. We assume that all orbits are nondegenerate. $m\left(\gamma_{x}^{t}\right)$ is the number of negative eigenvalues of $B-d^{2} W\left(\tilde{\gamma}_{x}^{t}\right) . O(1)$ is bounded uniformly in $h$.

Proof. We have already verified that the condition on $V$ allows us to apply the results of Sect. 4 in [1] (in particular Th. 4.1) to the oscillatory integral (4.3) for $K^{h}(t, x, x)$. This then yields, under the assumption of nondegeneracy, that the integral over $\mathscr{H}_{0,0}$ in (4.3) possesses an asymptotic expression in $h$, whose leading term is the one given in the lemma.

Remark 1 . In the case where the nondegeneracy condition is not satisfied, i.e. there are some closed orbits for which 0 is an eigenvalue of the operator $D\left(\gamma_{x}^{t}\right) \equiv$ $\left|\operatorname{Det} \frac{A}{\sin A t}\right|^{-1 / 2}\left(1-B^{-1} d^{2} W\left(\tilde{\gamma}_{x}^{t}\right)\right)$, then the asymptotic expression for $K^{h}(t, x, x)$ as $h \rightarrow 0$ is of the type

$$
K^{h}(t, x, x)=\sum_{\gamma_{x}^{\prime}} \exp \left(\frac{i}{h} S_{t}\left(\gamma_{x}^{t}\right)\right) G\left(h ; \gamma_{x}^{t}\right),
$$

where the sum is again over the closed orbits and the function $G\left(h ; \gamma_{x}^{t}\right)$ behaves as $h \rightarrow 0$ in a way which is uniquely determined by the type of singularity of the critical point of the phase, i.e. of the operator $D\left(\gamma_{x}^{t}\right)$. This is discussed in Sect. 5 of $[1]$.

Remark 2. Under the assumption on $V$ of Lemma 4.1 the potential $V$ is analytic and one can show that the set of degenerate critical points is discrete (see [27]). Moreover under the nondegeneracy assumption the set of closed orbits $\gamma_{x}^{t}$ is finite (see [27]).

Remark 3. Actually the results in Sect. 4 of [1] also give information about the higher order corrections to the leading term for $K^{h}(t, x, x)$ given in Lemma 4.1, in the case of nondegeneracy, in form of an asymptotic series in powers of $h$ for $O(1)$.

To compute the quantity $\left|D\left(\gamma_{x}^{t}\right)\right|$ appearing in Lemma 4.1, we consider following Truman [32] the Wiener integral

$$
E\left(\exp \left(\int_{0}^{t}(w(\tau), T(\tau) w(\tau))_{d} d \tau\right)\right),
$$

where $2 T(\tau)=A^{2}+d^{2} W(\tilde{\gamma}(\tau))$ and $\tilde{\gamma}=\gamma-\alpha(x)-\beta, \gamma$ being any of the closed orbits $\gamma_{x}^{t}$, the expectation being with respect to the conditional Wiener measure for paths $w$ in $\mathbb{R}^{d}$ starting at $\tau=0$ in $x=0$ and ending at $\tau=t$ in $x=0$. We assume first $T(\tau) \geqq 0$, which is e.g. satisfied for $t$ sufficiently small. Let $\gamma_{x, j}^{t}(\tau)$ be the $j^{\text {th }}$ component of $\gamma_{x}^{t}(\tau)$ in $\mathbb{R}^{d}$ and

$$
\left.p_{0, k} \equiv \frac{\partial \gamma_{x, k}^{t}(\tau)}{\partial \tau}\right|_{\tau=0} .
$$

It follows from a well known result of Cameron and Martin that (4.20) is equal to 


$$
\left|\operatorname{Det}\left(\left(\frac{\partial \gamma_{x, j}^{t}}{\partial p_{0, k}}\right)\right)\right|^{-1 / 2} \frac{1}{(2 \pi)^{d / 2}}
$$

where by $\frac{\partial \gamma_{x, j}^{t}}{\partial p_{0, k}}$ we understand the evaluation of $\frac{\partial \gamma_{x, j}^{t}(\tau)}{\partial p_{0, k}}$ at $\tau=t$. Comparing with the expression for the leading term of $K^{h}(t, x, x)$, we then get

$$
\left|D\left(\gamma_{x}^{t}\right)\right|^{1 / 2}=\left|\operatorname{Det}\left(\left(\frac{\partial \gamma_{x, j}^{t}}{\partial p_{0, k}}\right)\right)\right|^{1 / 2}
$$

By using a decomposition of the interval $[0, t]$ in small subintervals together with the Fubini theorem on iterated integrations one deduces that (4.22) holds also for arbitrary $t$. Using the above computation we see moreover that the index $m\left(\gamma_{x}^{t}\right)$ in Lemma 4.1 coincides with the number of negative eigenvalues of $\left(\left(\frac{\partial \gamma_{x, j}^{t}}{\partial p_{0, k}}\right)\right)$.

Hence $m\left(\gamma_{x}^{t}\right)$ is identified with Maslov's index for the closed orbit $\gamma_{x}^{t}$. This is independent of the point $x$ on the orbit (for concepts of Maslov's theory see e.g. [19], [25], [26], [33]). We formulate this result in the following

Lemma 4.2. With the same assumptions and notations as the Lemma 4.1 we have

$$
K^{h}(t, x, x)=\sum_{\gamma_{x}^{l}} \frac{\exp \left(\frac{i}{h} S_{t}\left(\gamma_{x}^{t}\right)\right) \exp \left(-\frac{i \pi}{2} m\left(\gamma_{x}^{t}\right)\right)}{\left|\operatorname{Det}\left(\left(\frac{\partial \gamma_{x, j}^{t}}{\partial p_{0, k}}\right)\right)\right|^{1 / 2}(2 \pi i h)^{d / 2}}(1+h O(1))
$$

where $\gamma_{x, j}^{t} j=1, \ldots, d$ is the $j^{\text {th }}$ component of the closed orbit $\gamma_{x}^{t}$ and $\left.p_{0, k} \equiv \frac{\partial \gamma_{x, j}^{t}(\tau)}{\partial \tau}\right|_{\tau=0}$ is the $k^{\mathrm{th}}$ component of the initial momentum on the orbit $\gamma_{x}^{t}, k=1, \ldots, d . m\left(\gamma_{x}^{t}\right)$ is the Maslov index of the closed orbit $\gamma_{x}^{t}$ and depends only on $\gamma_{x}^{t}$ but not on the initial point $x$ on $\gamma_{x}^{t}$.

Remark 4. If there are no closed orbits going through $x$ the sum above is understood in the sense that the leading term vanishes.

Remark 5. From this Lemma 4.2 we see that the condition of nondegeneracy of the closed orbit in Lemma 4.1 and in the corresponding remarks is actually the condition that the matrix $\left(\left(\frac{\partial \gamma_{x, j}^{t}}{\partial p_{0, k}}\right)\right)$ be nondegenerate.

In the expression for $K^{h}(t, x, x)$ in Lemma 4.2 the closed orbit $\gamma_{x}^{t}$ starting and ending at $x$ enters. Let us call two closed orbits $\gamma_{x}^{t}$ and $\gamma_{x}^{\prime t}$ equivalent if they coincide as subsets of $\mathbb{R}^{d}$. We shall denote by $\gamma$ the equivalence class (called again a closed orbit) with a representative $\gamma_{x}^{t}$. Let $\varphi$ be a $C_{0}^{\infty}\left(\mathbb{R}^{d}\right)$ function. Then from Lemma 4.2

$$
\int_{\mathbb{R}^{d}} K^{h}(t, x, x) \varphi(x) d x=\sum_{\mathscr{C}^{t}} \int_{R^{d}} \frac{\exp \left(\frac{i}{h} S_{t}\left(\gamma_{x}^{t}\right)\right)}{\operatorname{Det}\left(\left(\frac{\partial \gamma_{x, j}^{t}}{\partial p_{0, k}}\right)\right)^{1 / 2}(2 \pi i h)^{d / 2}}+h^{-d / 2+1} O(1),
$$

where $\mathscr{C}^{t}$ is the set of all closed orbits $\gamma$ (with representative $\gamma_{x}^{t}$ ) of period $t$. 
We shall now discuss the integral on the right hand side of (4.23) in the limit $h \rightarrow 0$ applying the method of stationary phase. The stationary points of the phase are given by $d_{x} S_{t}\left(\gamma_{x}^{t}\right)=0$. But

$$
\begin{aligned}
d_{x} S_{t}\left(\gamma_{x}^{t}\right) & =d_{x} \int_{0}^{t}\left[\frac{1}{2}\left(\dot{\gamma}_{x}^{t}(\tau), \dot{\gamma}_{x}^{t}(\tau)\right)_{d}-\frac{1}{2}\left(\gamma_{x}^{t}(\tau), A^{2} \gamma_{x}^{t}(\tau)\right)_{d}-V\left(\gamma_{x}^{t}(\tau)\right)\right] d \tau \\
& =\left.\left(\dot{\gamma}_{x}^{t}(\tau), d_{x} \gamma_{x}^{t}(\tau)\right)_{d}\right|_{0} ^{t}=p_{x}(t)-p_{x}(0),
\end{aligned}
$$

with $p_{x}(\tau) \equiv \dot{\gamma}_{x}^{t}(\tau)$ where we have performed a partial integration and used that $\gamma_{x}^{t}(\tau)$ is a closed orbit. We remark that $p_{x}(0)$ is the initial momentum we denoted before simply by $p_{0}$. From (4.24) we see that the stationary points are those for which $p_{x}(t)=p_{x}(0)$, hence the orbits which contribute to the leading term as $h \rightarrow 0$ are not only closed but periodic. We call orbits, i.e. solutions of $(4.18)$, for $\tau \in(0, t)$ periodic orbits (of period $t$, starting at $x$ ), if $\dot{\gamma}_{x}^{t}(t)=\dot{\gamma}_{x}^{t}(0)$. Let $\mathscr{P}^{t}$ be the set of all such periodic orbits.

We shall now derive the expressions for the leading term of (4.23) as $h \rightarrow 0$ by method of stationary phase in finite dimensions. We consider a point $x$ on a periodic orbit $\gamma_{x}^{t}$. In a neighbourhood $U_{x}$ of $x$ we introduce transversal $\left(x_{T}\right)$ and a longitudinal $\left(x_{L}\right)$ coordinates, with respect to the periodic orbit. Let $\tilde{\gamma}_{x}^{t}$ be a closed orbit starting and ending at 0 respectively $t$ in $x$. Consider $S_{t}\left(\tilde{\gamma}_{x}^{t}\right)$. We have $d_{x} S_{t}\left(\tilde{\gamma}_{x}^{t}\right)=\tilde{p}_{x}^{t}(t)-\tilde{p}_{x}^{t}(0)$, where $\tilde{p}_{x}^{t}(\tau) \equiv \dot{\gamma}_{x}^{t}(\tau)$, since $\tilde{\gamma}_{x}^{t}$ solves the classical equation of motion with $\tilde{\gamma}_{x}^{t}(t)=\tilde{\gamma}_{x}^{t}(0)=x$ (by the same computation as in (4.24)). We have $d_{x_{L} x_{L}} S_{t}\left(\tilde{\gamma}_{x}^{t}\right)=d_{x_{L}}\left(\tilde{p}_{x}^{t, L}(t)-\tilde{p}_{x}^{t, L}(0)\right)=\left.\frac{\partial}{\partial \tau} \tilde{p}_{x}^{L, t}(\tau) \frac{1}{\dot{\gamma}(\tau)}\right|_{0} ^{t}$, where $\tilde{p}_{x}^{t, L}(\tau)$ is the longitudinal component of $\tilde{p}_{x}^{t}(\tau)$ and this is zero for $\tilde{\gamma}_{x}^{t}=\gamma_{x}^{t}$, using the classical equation of motion $\left.\frac{\partial}{\partial \tau} \tilde{p}_{x}^{t}(\tau)\right|_{\tau=t}=-\nabla V(x)-A^{2} x=\left.\frac{\partial}{\partial \tau} \tilde{p}_{x}^{t}(\tau)\right|_{\tau=0}$ and the fact that for a periodic orbit one has $\dot{\gamma}_{x}(t)=\dot{\gamma}_{x}(0)$. Furthermore $d_{x_{T} x_{L}} S_{t}\left(\tilde{\gamma}_{x}^{t}\right)=d_{x_{T}}\left(\tilde{p}_{x}^{t, L}(t)-\tilde{p}_{x}^{t, L}(0)\right)=0$ for $\tilde{\gamma}_{x}^{t}=\gamma_{x}^{t}$. Finally $d_{x_{T} x_{T}} S_{t}\left(\tilde{\gamma}_{x}^{t}\right)=d_{x_{T}}\left(\tilde{p}_{x}^{t, T}(t)-\tilde{p}_{x}^{t, T}(0)\right)$, where $\tilde{p}_{x}^{t, T}(\tau)$ is the transversal component of $\tilde{p}_{x}^{t}(\tau)$. Thus

$$
d_{x x} S_{t}\left(\tilde{\gamma}_{x}^{t}\right) \mid \tilde{\gamma}_{\gamma_{x}^{t}=\gamma_{x}^{t}}=\left(\begin{array}{ll}
a & 0 \\
0 & 0
\end{array}\right), \quad \text { with } a \equiv d_{x_{T}}\left(p_{x}^{t, T}(t)-p_{x}^{t, T}(0)\right)
$$

where $d_{x_{T}}\left(p_{x}^{t, T}(t)-p_{x}^{t, T}(0)\right)$ is the value at $\tilde{\gamma}_{x}^{t}=\gamma_{x}^{t}$ of $d_{x_{T} x_{T}} S_{t}\left(\tilde{\gamma}_{x}^{t}\right)$. In particular $d_{x x} S_{t}\left(\gamma_{x}^{t}\right)$ is independent of $x_{L}$. We remark that the nonvanishing term in (4.25) is of the form $R(t)-1$, where $R(t)$ is the part of the Poincare map for the classical Hamiltonian flow relative to the transverse momenta.

We shall now apply the method of stationary phase to (4.23), splitting the integration over $d x$ in an integration over $d x_{T}$ first and then over $d x_{L}$. By a partition of the unit we see that we can always suppose that $\varphi$ has support in $U_{x}$, the remainder giving only a contribution vanishing faster than any power of $h$ as $h \rightarrow 0$. In this way we obtain, using (4.22):

$$
\int \frac{\exp \left(\frac{i}{h} S_{t}\left(\gamma_{x}^{t}\right)\right) \exp \left(-i \frac{\pi}{2} m\left(\gamma_{x}^{t}\right)\right)}{\mid \operatorname{Det}\left(\left.\left(\frac{\partial \gamma_{x, i}^{t}}{\partial p_{0, k}}\right)\right|^{1 / 2}\right.} \frac{\varphi(x)}{(2 \pi i h)^{d / 2}} d x
$$




$$
\begin{gathered}
=\int_{\gamma_{x}^{t}}(2 \pi i h)^{\frac{d-1}{2}} \frac{\exp \left(\frac{i}{h} S_{t}\left(\gamma_{x}^{t}\right)\right) \exp \left(-i \frac{\pi}{2} m\left(\gamma_{x}^{t}\right)\right)}{\left|\Delta\left(\gamma_{x}^{t}\right)\right|^{1 / 2}\left|D\left(\gamma_{x}^{t}\right)\right|^{1 / 2}(2 \pi i h)^{d / 2}} \\
\cdot \exp \left(-i \frac{\pi}{2} \tilde{m}\left(\gamma_{x}^{t}\right)\right) \varphi\left(x_{L}, 0\right) d x_{L}\{1+h O(1)\},
\end{gathered}
$$

where $\Delta\left(\gamma_{x}^{t}\right)=\operatorname{Det} d_{x_{T}}\left(p_{x}^{T}(t)-p_{x}^{T}(0)\right)$, computed for $\gamma \in \mathscr{P}^{t}$.

Here $\tilde{m}$ is the number of negative eigenvalues of the $(d-1) \times(d-1)$ matrix $d_{x_{T}}\left(p_{x}^{T}(t)-p_{x}^{T}(0)\right)$. But on the other hand $S_{t}\left(\gamma_{x}^{t}\right)$ and $m\left(\gamma_{x}^{t}\right)$ are independent of $x_{L}$, for $\gamma \in \mathscr{P}^{t}$, and we write them simply as $S_{t}(\gamma)$ respectively $m(\gamma)$. Taking this into account we get that the asymptotic evaluation for $h \rightarrow 0$ of (4.23) is

$$
\int K^{h}(t, x, x) \varphi(x) d x=\sum_{\mathscr{P} t} \frac{\exp \left(\frac{i}{h} S_{t}(\gamma)\right)}{(2 \pi i h)^{1 / 2}} \exp \left(-i \frac{\pi}{2} m^{\prime}(\gamma)\right) A_{\varphi}(\gamma)\{1+h O(1)\},
$$

where $A_{\varphi}(\gamma)$ is a number depending on the periodic orbit $\gamma$ and on $\varphi$ :

$$
A_{\varphi}(\gamma)=\int_{\gamma}\left|\Delta\left(\gamma_{x}^{t}\right)\right|^{-1 / 2}\left|D\left(\gamma_{x}^{t}\right)\right|^{-1 / 2} \varphi\left(x_{L}, 0\right) d x
$$

and $m^{\prime}(\gamma) \equiv m(\gamma)+\tilde{m}(\gamma)$.

We say that a periodic orbit $\gamma \in \mathscr{P}^{t}$ has primitive period $t$ if there does not exist any positive integer $n$ such that $t / n$ is a period of $\gamma$. If a periodic orbit $\gamma$ of period $t$ does not have primitive period $t$ then there exists an $n$ and a primitive periodic orbit $\gamma^{t / n}$ of period $t / n$ such that $\gamma$ is $\gamma^{t / n}$ run $n$ times. Let $\mathscr{P}_{p}^{t / n}$ be the family of all primitive periodic orbits of period $t / n$. We can then split the sum over $\mathscr{P P}^{t}$ in (4.27) as $\sum_{n} \sum_{\cot ^{t / n}}$. Let for $\gamma \in \mathscr{P}^{t}, \gamma^{t / n}$ be the primitive periodic orbit such that $\gamma$ is $\gamma^{t / n}$ run $n$ times. Then $S_{t}(\gamma)=n S_{t / n}\left(\gamma^{t / n}\right)$ and $m(\gamma)=n m\left(\gamma^{t / n}\right), \tilde{m}(\gamma)=n \tilde{m}\left(\gamma^{t / n}\right)$, thus $m^{\prime}(\gamma)=$ $n m^{\prime}\left(\gamma^{t / n}\right)$, and the formula (4.27) can be rewritten in the form

$$
\begin{aligned}
\int K^{h}(t, x, x) \varphi(x) d x= & \sum_{n=1}^{\infty} \sum_{\mathscr{P}_{t}^{t / n}} \frac{\exp \left(\frac{i}{h} n S_{t / n}\left(\gamma^{t / n}\right)\right)}{(2 \pi i h)^{1 / 2}} \exp \left(-i \frac{\pi}{2} n m^{\prime}\left(\gamma^{t / n}\right)\right) \\
& \cdot A_{\varphi}\left(\gamma^{t / n}\right)\{1+h O(1)\} .
\end{aligned}
$$

We now remark that the potential $V$ being smooth by our assumptions, the Hamiltonian function $\frac{1}{2} \dot{\gamma}(\tau)^{2}+\frac{1}{2}\left(\gamma(\tau), A^{2} \gamma(\tau)\right)_{d}+V(\gamma(\tau))$ associated with a classical orbit $\gamma(\tau)$ is constant in time. In particular for $\gamma$ a periodic orbit of period $t$ it is equal to $E_{t}^{\gamma}$, where $E_{t}^{\gamma}$ is one of the possible energies corresponding to the prescribed period $t$.

We now suppose that $\left|E_{t}^{\gamma}\right|<C_{t}$ for all $\gamma \in \mathscr{P} t$, for some $C_{t}$ independent of $\gamma$. Then $|\dot{\gamma}(\tau)|<C_{t}^{1},|\gamma(\tau)|<C_{t}^{2}$ for all $\tau \in[0, t]$ and some $C_{t}^{1}, C_{t}^{2}$ independent of $\gamma \in \mathscr{P}^{t}$. Taking $\varphi=\varphi_{R, \delta}$ in (4.29) equal 1 on the sphere in $\mathbb{R}^{d}$ of radius $R>C_{0}^{t}$ and center at 0 , and satisfying $0 \leqq \varphi \leqq 1$ and moreover $\varphi=0$ outside the sphere of radius $R+\delta, \delta>0$, with center at 0 , we have (4.29) with $A \varphi\left(\gamma^{t / n}\right)$ replaced by 


$$
A\left(\gamma^{t / n}\right) \equiv \int_{\gamma}\left|\Delta\left(\gamma_{x}^{t}\right)\right|^{-1 / 2}\left|D\left(\gamma_{x}^{t}\right)\right|^{-1 / 2} d x
$$

For $t$ outside a discrete set (where $K_{0}^{h}(t, x, x)$ is singular) we can for given $\varepsilon>0$ find a $\delta(\varepsilon)$ such that $\int_{|x| \geqq R} K^{h}(t, x, x) \varphi_{R, \delta}(x) d x<\varepsilon$, hence

$$
\left|\int_{|x|<R} K^{h}(t, x, x) d x-K^{h}(t, x, x) \varphi_{R, \delta}(x) d x\right|<\varepsilon .
$$

Hence for the $t$ such that $\int K^{h}(t, x, x) d x$ exists as a limit of $\int_{|x|<R} K^{h}(t, x, x) d x$ for $R \rightarrow \infty$ we can for given $\varepsilon>0$ choose $R(\varepsilon)$ and $\delta(\varepsilon)$ such that $\left|\int K^{h}(t, s, s) d x-\int K^{h}(t, s, s) \varphi_{R, \delta}(x) d x\right|<\varepsilon$. Thus $\int K^{h}(t, x, x) d x$ is given by the right hand side of (4.29) with $A_{\varphi}\left(\gamma^{t / n}\right)$ replaced by (4.30). We summarize these results in the following

Theorem 4.3. Let $V \in \mathbb{F}\left(\mathbb{R}^{d}\right)$ such that $\int_{\mathbb{R}} e^{\varepsilon|\alpha|} d\left|\mu_{V}\right|(\alpha)<\infty$ for some $\varepsilon>0$ and let all periodic orbits of period $t$ be nondegenerate. Then for any $\varphi \in C_{0}^{\infty}\left(\mathbb{R}^{d}\right)$

$$
\begin{aligned}
\int K^{h}(t, x, x) \varphi(x) d x= & \sum_{n=1}^{\infty} \sum_{\substack{\mathscr{P}_{p}^{t / n}\\
}} \frac{\exp \left(\frac{i}{h} n S_{t / n}\left(\gamma^{t / n}\right)\right)}{(2 \pi i h)^{1 / 2}} \exp \left(-i \frac{\pi}{2} n m^{\prime}\left(\gamma^{t / n}\right)\right) \\
& \cdot A_{\varphi}\left(\gamma^{t / n}\right)\{1+h O(1)\},
\end{aligned}
$$

with $\gamma_{p}^{t / n}$ the family of all primitive periodic orbits of period $t / n$, and $m^{\prime}\left(\gamma^{t / n}\right)$ a Maslov index for $\gamma^{t / n}$. This holds also for $\varphi \equiv 1$ for all $t$ such that $\theta_{h}(t) \equiv \int K^{h}(t, s, s) d x$ exists, with $A_{\varphi}\left(\gamma^{t / n}\right)$ then given by

$$
A\left(\gamma^{t / n}\right) \equiv \int_{\gamma}\left|\Delta\left(\gamma_{x}^{t}\right)\right|^{-1 / 2}\left|D\left(\gamma_{x}^{t}\right)\right|^{-1 / 2} d x
$$

The quantity $A_{\varphi}(\gamma)$ involves $D\left(\gamma_{x}^{t}\right)$, which is given by (4.22). It is known from classical mechanics that

$$
\left(\left(\frac{\partial \gamma_{i}^{t}}{\partial p_{x, j}(0)}\right)\right)=\left(\left(\frac{\partial^{2} S_{t}\left(\gamma^{t}\right)}{\partial x_{i}(t) \partial x_{j}(0)}\right)\right)^{-1} \quad i, j=1, \ldots, d .
$$

Let us now consider the Hamiltonian system $(q(\tau), p(\tau))$ with the single energy integral $E$. Let $S_{\tau}$ be the Hamilton-Jacobi function $S_{\tau}(y, \tau ; x, 0)$. Then one has

$$
\left.\frac{\partial S_{\tau}}{\partial \tau}\right|_{\tau=t}+E=0
$$

when $t=t(x, y, E)$ is the time for a classical path going from $x$ to $y$. Let $W_{E}$ be the Legendre transform of $S_{t}$, i.e.

$$
W_{E}=S_{t}+t E .
$$

It is then easy to show that

$$
\frac{\partial^{2} S}{\partial t^{2}}=-\left(\frac{\partial^{2} W}{\partial E^{2}}\right)^{-1} \text { and } \frac{\partial E}{\partial y_{j}}=-\frac{\partial^{2} W_{E}}{\partial y_{j} \partial E} / \frac{\partial^{2} W}{\partial E^{2}} .
$$


So one gets

$$
\frac{\partial^{2} S_{t}}{\partial x_{i} \partial y_{j}}=-\frac{\partial^{2} W_{E}}{\partial y_{j} \partial E} \frac{\partial^{2} W_{E}}{\partial x_{i} \partial E} \mid \frac{\partial^{2} W_{E}}{\partial E^{2}}
$$

where we used the Hamilton equations. Assume now that to given periods $t^{\prime}$ in a neighbourhood of the given period $t$ there exist periodic orbits with energy $E^{\prime}$ such that $\left.\frac{d t^{\prime}}{d E^{\prime}}\right|_{t^{\prime}=t}$ is well defined. Observing that $\frac{\partial W_{E}}{\partial E}=\frac{d t}{d E}$, we obtain the following representation

$$
\left(\left(\frac{\partial \gamma_{j}^{t}}{\partial p_{x_{i}}(0)}\right)\right)=-\frac{d t}{d E}\left(\left(\frac{\partial t}{\partial x_{j}}, \frac{\partial t}{\partial x_{i}}\right)\right)
$$

Furthermore we have, introducing $x_{T}, x_{L}$ as variables:

$$
\left(\left(\frac{\partial t}{\partial x_{j}}, \frac{\partial t}{\partial x_{i}}\right)\right)=\left(\left(\begin{array}{cc}
\dot{\gamma}^{t}(0)^{2} & 0 \\
0 & \frac{\partial^{2} t}{\partial x_{T} \partial x_{T}}
\end{array}\right)\right) .
$$

The matrix $D_{T} \equiv\left(\left(\frac{\partial^{2} t}{\partial x_{T} \partial x_{T}}\right)\right)$ is independent of $x_{L}$. Then the quantity $\left|D\left(\gamma_{x}^{t}\right)\right|^{-1 / 2}$ entering $A_{\varphi}(\gamma)$ in (4.28) is equal to $\left|\frac{d t}{d E}\right|^{1 / 2}\left|\dot{\gamma}_{x}^{t}(0)\right| /\left|D_{T}\right|^{1 / 2}$, where $\left|D_{T}\right|$ is the determinant of $D_{T}$. Moreover, along $\gamma, d x_{L}=\left|\dot{\gamma}_{x}^{t}(0)\right| d \tau$, hence

$$
A_{\varphi}(\gamma)=\frac{1}{\left|D_{T}\right|^{1 / 2}}\left|\frac{d E}{d t}\right|^{1 / 2} \frac{\int_{0}^{t} \varphi\left(\gamma_{x}^{t}(\tau), 0\right) d \tau}{\left|\operatorname{Det} d_{x_{T}}\left(p_{x}(t)-p_{x}(0)\right)\right|^{1 / 2}} .
$$

Now because of the above interpretation of $d_{x_{T}}\left(p_{x}(t)-p_{x}(0)\right)$ we have from classical mechanics that its determinant has the form

$$
\operatorname{Det}\left(d_{x_{T}}\left(p_{x}(t)-p_{x}(0)\right)\right)=\prod_{k=1}^{d-1}\left(4 \cos ^{2} i \beta_{k} t\right)
$$

where the $\beta_{k}$ are numbers, the stability angles (see [16]). Thus

Define

$$
A_{\varphi}(\gamma) \equiv \frac{1}{\left|D_{T}\right|^{1 / 2}}\left|\frac{d E}{d t}\right|^{1 / 2}\left|\prod_{k=1}^{d-1} 4 \cos ^{2} i \beta_{k}\right|^{-1 / 2} \int_{0}^{t} \varphi\left(\gamma_{x}^{t}(\tau), 0\right) d \tau .
$$

$$
A(\gamma) \equiv \frac{t}{\left|D_{T}\right|^{1 / 2}}\left|\frac{d E}{d t}\right|^{1 / 2}\left|\prod_{k=1}^{d-1} 4 \cos ^{2} i \beta_{k} t\right|^{-1 / 2}
$$

(so that $A(\gamma)$ is $A_{\varphi}(\gamma)$ for $\varphi \equiv 1$ ).

We have then the formulae of Theorem 4.3 with $A \varphi\left(\gamma^{t / n}\right)$ and $A\left(\gamma^{t / n}\right)$ given by (4.33) respectively (4.34).

Finally we examine the case $d=1$. In this case there are no transversal coordinates, hence the right hand side of (4.32) as well as $D_{T}$ are to be taken equal to 1 . 
Thus in this case

and

$$
A_{\varphi}\left(\gamma^{t / n}\right)=\sqrt{n}\left|\frac{d E}{d t}\left(\gamma^{t / n}\right)\right| \int_{0}^{1 / 2 t / n} \varphi\left(\gamma_{x}^{t}(\tau), 0\right) d \tau
$$

$$
A\left(\gamma^{t / n}\right)=\frac{t}{\sqrt{n}}\left|\frac{d E\left(\gamma^{t / n}\right)}{d t}\right|^{1 / 2}
$$

We summarize these results in the following

Theorem 4.4. Let $V$ be as in Theorem 4.3. Then in the case where the energy is the only integral for the Hamiltonian system and to given periods $t^{\prime}$ in a neighborhood of the given time $t$ there exist periodic orbits with energy $E^{\prime}$ such that $\left.\frac{d t^{\prime}}{d E^{\prime}}\right|_{t^{\prime}=t} i s$ well defined, then for any $\varphi \in C_{0}^{\infty}\left(\mathbb{R}^{d}\right)$ :

and

$$
\begin{array}{r}
\int K^{h}(t, x, x) \varphi(x) d x=\sum_{n=1}^{\infty} \sum_{\mathscr{P}_{p}^{t / n}} \frac{\exp \left(\frac{i}{h} n S_{t / n}\left(\gamma^{t / n}\right)\right)}{(2 \pi i h)^{1 / 2}} \exp \left(-i \frac{\pi}{2} n m^{\prime}\left(\gamma^{t / n}\right)\right) C_{t / n} \\
\cdot \sqrt{n}\left|\frac{d E\left(\gamma^{t / n}\right)}{d t}\right|^{1 / 2} \int_{0}^{t / n} \varphi\left(\gamma_{x}^{t}(\tau), 0\right) d \tau\{1+h O(1)\} .
\end{array}
$$

$$
\begin{aligned}
\theta_{h}(t)= & \sum_{n=1}^{\infty} \sum_{\substack{\beta_{t / n}\\
}} \frac{\exp \left(\frac{i}{h} n S_{t / n}\left(\gamma^{t / n}\right)\right)}{(2 \pi i h)^{1 / 2}} \exp \left(-i \frac{\pi}{2} n m^{\prime}\left(\gamma^{t / n}\right)\right) C_{t / n} \\
& \frac{t}{\sqrt{n}}\left|\frac{d E\left(\gamma^{t / n}\right)}{d t}\right|^{1 / 2}(1+h O(1)),
\end{aligned}
$$

with $C_{t / n} \equiv\left|D_{T}\right|^{-1 / 2}\left|\prod_{k=1}^{d-1} 4 \cos ^{2} i \beta_{k} t / n\right|^{-1 / 2}$.

In the case $d=1$ we have $C_{t / n} \equiv 1$.

Remark. Theorem 4.3 and 4.4 give asymptotic (as $h \rightarrow 0$ ) formulae for the theta function in terms of classical periodic orbits and correspond thus to the known trace or Poisson formulae in the case of elliptic operators on compact manifolds.

In the case of the Laplacian on the torus $T=\mathbb{R}^{d} / \Gamma, \Gamma$ a discrete subgroup, the homogenity of the action $S_{t}(\gamma)=\frac{1}{2} \int_{0}^{t} \dot{\gamma}^{2}(\tau) d \tau$, gives an exact (the classical Poisson formula for the Jacobi theta function) instead of an asymptotic formula. Some information on the structure of classical periodic orbits can be extracted from [8].

\section{The Eigenvalue Spectrum and the $\theta$-Function}

In Sect. 3 we defined the $\theta$-function for the Schrödinger operator $H$ as the generalized function 


$$
\theta_{h}(t)=\operatorname{Tr} \exp \left(-\frac{i}{h} t H\right)=\sum_{n} \exp \left(-\frac{i}{h} t \hat{\lambda}_{n}(h)\right),
$$

where $\lambda_{n}(h)$ are the eigenvalues of the Schrödinger operator $H$. We have

$$
\theta_{h}(t)=\mathscr{F}\left[\sum_{n} \delta\left(\lambda-\frac{1}{h} \lambda_{n}(h)\right)\right](t),
$$

where $\mathscr{F}$ means the Fourier transform and $\sum_{n} \delta\left(\lambda-\frac{1}{h} \lambda_{n}(h)\right)$ is the spectral distribution. Since in our case the potential $V$ is bounded and continuous we have that $N\left(\frac{1}{h} \lambda_{n}(h) \leqq \lambda\right)$ has polynomial growth in $\lambda$, where $N(\alpha \leqq \beta)$ is the number of eigenvalues $\alpha$ of $H$ which are less or equal to $\beta$, so that $\theta_{h}$ is a tempered distribution.

It is now interesting to study the singularities of the theta function $\theta_{h}(t)$. Let us take a function $g$ such that $\int \theta_{h}(t) g(t) d t$ is defined in the sense of generalized functions. We first observe that if the Fourier transform $\hat{g}(\lambda)$ of $g(t)$ exists and has support around $\frac{1}{h} \lambda_{n}(h)$, where $\lambda_{n}(h)$ is an eigenvalue of $H$, such that supp $\hat{g}$ contains no point $\frac{1}{h} \lambda_{n^{\prime}},(h), n^{\prime} \neq n$, then we have

$$
\hat{g}\left(\frac{1}{h} \lambda_{n}(h)\right)=\int \theta_{h}(t) g(t) d t \equiv\left\langle g, \theta_{h}\right\rangle .
$$

In the following we find it more convenient to consider functions $g$ of the form $g_{\lambda}(t)=\exp \left(\frac{i}{h} \lambda t\right) f(t)$, where $f(t)$ is a real function in $\mathscr{S}\left(\mathbb{R}_{+}\right)$such that $\left\langle g_{\lambda}, \theta_{h}\right\rangle$ exists and $f(t)=0$ for $t \leqq 0$. The method of Sect. 4 gives an asymptotic expansion for $\theta_{h}(t)$ as $h \rightarrow 0$. In particular we have

$$
\theta_{h}(t)=\theta_{h}^{0}(t)+h^{\frac{1}{2}} O_{t}(1)
$$

where the leading term $\theta_{h}^{o}(t)$ has been given in Theorem 4.3 respectively 4.4 .

Observing that $O_{t}(1)$ is slowly increasing in $t$, as follows easily from our asymptotic expansions (see for the higher order terms the theorem in Sect. 4 of [1]) we have

$$
\left\langle g_{\lambda}, \theta_{h}\right\rangle=\left\langle g_{\lambda}, \theta_{h}^{0}\right\rangle+h^{\frac{1}{2}} O(1)
$$

Now the expression for $\theta_{h}^{O}(t)$ given by Theorem 4.3 and Theorem 4.4 is

$$
\begin{gathered}
\theta_{h}^{0}(t)=\sum_{n=1}^{\infty} \sum_{\lambda^{t / n}} \frac{\exp \left(\frac{i}{h} n S_{t / n}\left(\gamma^{t / n}\right)\right)}{(2 \pi i h)^{d / 2}} \exp \left(-i \frac{\pi}{2} n m^{\prime}\left(\gamma^{t / n}\right)\right) A\left(\gamma^{t / n}\right) \\
\equiv \sum_{n=1}^{\infty} \sum_{\gamma^{t / n}} \check{\theta}_{h}\left(\gamma^{t / n}\right) .
\end{gathered}
$$

We now want to apply to $\left\langle g_{\lambda}(t), \check{\theta}_{h}\left(\gamma^{t / n}\right)\right\rangle$ the method of stationary phase with respect to the $t$-integration. We assume that $g_{\lambda}$ has compact support. Let $\mathscr{P}_{n}^{t}$ be 
the family of periodic orbits $\gamma^{t / n}$ of period $t$ and primitive period $t / n$. Let $\mathscr{P}_{\lambda, n}=\bigcup_{t \in \operatorname{supp} g_{\lambda}} \mathscr{P}_{n}^{t}$. We suppose that $\mathscr{P}_{\lambda, n}$ consists of families of periodic orbits $\left(\gamma^{t / n}, t \in \operatorname{supp} g_{\lambda}\right)$ such that the following properties hold:

a) $S_{t / n}\left(\gamma^{t / n}\right)$ is $C^{2}$ in $t$ for all $t$ in the inner $\operatorname{Int}\left(\operatorname{supp} g_{\lambda}\right)$ of $\operatorname{supp} g_{\lambda}$,

b) $\frac{\partial^{2} S_{t / n}}{\partial t^{2}}\left(\gamma^{t / n}\right) \neq 0$ for all $t \in \operatorname{Int}\left(\operatorname{supp} g_{\lambda}\right)$.

Now the stationary points of the phase of the integral $\left\langle g_{\lambda}(t), \check{\theta}_{h}\left(\gamma^{t / n}\right)\right\rangle$ are the solutions $t(\lambda)$ of the equation

$$
n \frac{\partial S_{t / n}}{\partial t}\left(\gamma^{t / n}\right)+\lambda=0
$$

for given $\lambda \in \mathbb{R}$. It is well known from analytical mechanics that for a classical orbit $\tilde{\gamma}$ and action $S_{\tau}$ the relation between time $\tau$ and energy $E$ is given by

$$
\frac{\partial S_{\tau}}{\partial \tau}(\tilde{\gamma})+E=0
$$

$E$ is thus the energy associated with the classical orbit $\tilde{\gamma}$ and the action function $S_{\tau}(\tilde{\gamma})$ (in the time interval $[0, \tau]$ ). From $(5.8)$ and (5.9) we see that the parameter $\lambda$ plays the role of energy parameter for the orbit $\gamma^{t / n}$ of primitive period $t / n$ run in time $t / n$, i.e. with $\tau=t / n, \tilde{\gamma}=\gamma^{t / n}$ we have that (5.8) is equal (5.9), with $\lambda=E$. I.e. the values of $\lambda$ for which there are indeed classical periodic orbits can be interpreted as energy values.

The method of stationary phase gives now under the above assumptions, with

$$
\begin{aligned}
& \lambda=E \text { and }\left(\gamma^{t / n}, t \in \operatorname{supp} g_{E}\right) \in \mathscr{P}_{E, n}, \\
& \left\langle g_{E}(t), \check{\theta}_{h}\left(\gamma^{t / n}\right)\right\rangle= \\
& \sum_{t(E)} f(t(E)) \frac{\exp \left(\frac{i}{h} n S_{t(E) / n}\left(\gamma^{t(E) / n}\right)\right)}{(2 \pi i h)^{d / 2}}(2 \pi i h)^{1 / 2} \exp \left(\frac{i}{h} E t(E)\right) \\
& \cdot \exp \left(-i n \frac{\pi}{2} \tilde{m}\left(\gamma^{t(E) / n}\right)\right) \frac{A\left(\gamma^{t(E) / n}\right)}{\left|\Delta\left(\gamma^{t(E) / n}\right)\right|^{1 / 2}}+h^{1 / 2} O(1),
\end{aligned}
$$

where the sum is over all values of $t(E)$ in supp $g_{E}$ such that (5.9) is satisfied for the periodic orbit $\gamma^{t(E) / n}$ of period $t(E)$ and primitive period $t(E) / n$. We make as before the convention that the sum is zero if the set of such $t(E)$ is void. The quantity $\tilde{m}$ is defined by

$$
\tilde{m}\left(\gamma^{t(E) / n}\right) \equiv m\left(\gamma^{t(E) / n}\right)+\sigma\left(\gamma^{t(E) / n}\right),
$$

where $\quad \sigma\left(\gamma^{t(E) / n}\right) \equiv 0$ if $\left.\frac{\partial^{2} S}{\partial \tau^{2}}\left(\gamma^{t / n}\right)\right|_{\tau=t(E)}>0 \quad$ and $\sigma\left(\gamma^{t(E) / n}\right) \equiv 2$ if $\left.\frac{\partial^{2} S}{\partial \tau^{2}}\left(\gamma^{\tau / n}\right)\right|_{\tau=t(E)}<0$. The quantity $\left|\Delta\left(\gamma^{t(E) / n}\right)\right|$ is defined by

$$
\left.\left|\Delta\left(\gamma^{t(E) / n}\right) \equiv \frac{1}{n}\right| \frac{\partial^{2} S_{\tau / n}}{\partial \tau^{2}}\left(\gamma^{\tau / n}\right)\right|_{\tau=t(E)} .
$$


We note that if $t(E)$ is a solution of (5.9) for given $E$ then also $k t(E)$ is a solution, for all $k=1,2, \ldots$. Moreover the orbit of period $k t(E)$ has primitive period $t(E) / n$ iff the orbit of period $t(E)$ has primitive period $t(E) / n$. Consider now

$$
\left\langle g_{E}(t), \sum_{n} \check{\theta}_{h}\left(\gamma^{t / n}\right)\right\rangle
$$

By the fact that the set of $n$ such that $t / n$ is a primitive period for some $t \in \operatorname{supp} g_{E}$ is finite, we have that (5.13) equals

$$
\sum_{n}\left\langle g_{E}(t) \check{\theta}_{h}\left(\gamma^{t / n}\right)\right\rangle
$$

Inserting now (5.10) into (5.4) we have a double sum $\sum_{n} \sum_{t(E)}$. This sum is over all periods $t(E) \in \operatorname{supp} g_{E}$ corresponding to (5.9) and such that $t(E) / n$ is for some $n$ a primitive period and $t(E) / n \in \operatorname{supp} g_{E}$.

Hence $\sum_{n} \sum_{t(E)}=\sum_{n} \sum_{t_{0}(E)}$ (setting first $t(E)=n t_{0}(E)$ and letting then $t_{n}(E)$ and $n$ vary) where $\sum_{t_{0}(E)}$ is the sum over all primitive periods $t_{0}(E) \in \operatorname{supp} g_{E}$ and $\sum_{n}$ is the sum over all such $n \in \mathbb{N}$ such that $n t_{0}(E) \in \operatorname{supp} g_{E}$, so that the sum on the right hand side can be interpreted as the sum over all periodic orbits with energy $E$, run $n$ times, provided $n t_{0}(E) \in \operatorname{supp} g_{E}, t_{0}(E)$ being the primitive period for the orbit in question.

Introducing this into (5.14) and using (5.14), (5.10) we get

$$
\begin{aligned}
& \left\langle g_{E}(t), \sum_{n} \check{\theta}_{h}\left(\gamma^{t / n}\right)\right\rangle=\sum_{n} \sum_{t_{0}(E)} f\left(n t_{0}(E)\right)\left((2 \pi i h)^{-(d-1) / 2} \exp \left(\frac{i}{h} n S_{t_{0}(E)}\left(\gamma^{t_{0}(E)}\right)\right)\right. \\
& \cdot \exp \left(\frac{i}{h} n E t_{0}(E)\right) \exp \left(-i n \frac{\pi}{2} \tilde{m}\left(\gamma^{t_{0}(E)}\right)\right) \frac{\tilde{A}\left(\gamma^{t_{0}(E)}\right)}{\left|\Delta\left(\gamma^{t_{0}(E)}\right)\right|^{1 / 2}}(1+h O(1)) .
\end{aligned}
$$

Let now $W_{E}$ be the Legendre transform of the action functional $S_{\tau}$ along a classical orbit in a time $t$ interval $\tau$, i.e.

$$
W_{E}=S_{\tau}+E \tau \text {. }
$$

Introducing (5.16) into (5.15) with $\tau=t_{0}(E)$, we get

$$
\begin{aligned}
& \left\langle g_{E}(t), \sum_{n} \check{\theta}_{h}\left(\gamma^{t / n}\right)\right\rangle=\sum_{n} \sum_{t_{0}(E)} f\left(n t_{0}(E)\right) \frac{\exp \left(\frac{i}{h} n W_{E}\left(\gamma^{t_{0}(E)}\right)\right)}{(2 \pi i h)^{(d-1) / 2}} \\
& \cdot \exp \left(-i n \frac{\pi}{2} \tilde{m}\left(\gamma^{t_{0}(E)}\right)\right) \frac{A\left(\gamma^{t_{0}(E)}\right)}{\left|\Delta\left(\gamma^{t_{0}(E)}\right)\right|^{1 / 2}}(1+h O(1)) .
\end{aligned}
$$

We can now compute $\left|\Delta\left(\gamma^{t_{0}(E)}\right)\right|^{1 / 2}$ to be equal to $n^{1 / 2}\left|\frac{d t_{0}(E)}{d E}\right|^{1 / 2}$. 
Hence from (5.17) and (5.6), (5.7) we get, observing that the sum in (5.7) is over $\mathscr{P P}_{n}^{t}$

$$
\begin{gathered}
\left\langle g_{E}, \theta_{h}\right\rangle=\sum_{n} \sum_{\Gamma(E)} f\left(n t_{0}(E)\right) \frac{\exp \left(\frac{i}{h} n W_{E}\left(\gamma^{t_{0}(E)}\right)\right)}{(2 \pi i h)^{(d-1) / 2}} \exp \left(-i n \frac{\pi}{2} \tilde{m}\left(\gamma^{t_{0}(E)}\right)\right) \\
\cdot n^{1 / 2}\left|\frac{d t_{0}(t)}{d E}\right|^{1 / 2} A\left(\gamma^{t_{0}(E)}\right)(1+h O(1)),
\end{gathered}
$$

where the sum $\sum_{\Gamma(E)}$ is over the class $\Gamma(E)$ consisting of all pairs $\left(t_{0}(E), \gamma^{t_{0}(E)}\right)$ such that $\gamma^{t_{0}(E)}$ is a periodic orbit of primitive period $t_{0}(E)$ and energy $E$. The sum over $n$ is restricted to those $n$ such that $n t_{0}(E) \in \operatorname{supp} g_{E}$.

Hence we have prove the following

Theorem 5.1. Let $V$ be as in Theorems 4.4 or 4.5. Let $E$ be a positive number and let $g_{E}(t)=\exp \left(\frac{i}{h} E t\right) f(t)$ with $f \in \mathscr{S}\left(\mathbb{R}_{+}\right)$and supp $f$ compact. Suppose supp $f$ is such that one can find some family $\left(\gamma^{t}, t \in \operatorname{supp} f\right)$ of periodic orbits $\gamma^{t}$ of primitive period $t$, where $t$ ranges over supp $f$. Suppose $S_{t}\left(\gamma^{t}\right)$ is $C^{2}$ in $t$ and nondegenerate in the inner of supp $f$. Then

$$
\begin{aligned}
\left\langle g_{E}, \theta_{h}\right\rangle & \left.=\sum_{n} \sum_{\Gamma^{(E)}} f(n) t(E)\right) \frac{\exp \left(\frac{i}{h} n W_{E}\left(\gamma^{t(E)}\right)\right)}{(2 \pi i h)^{(d-1) / 2}} \exp \left(-i n \frac{\pi}{2} \tilde{m}\left(\gamma^{t(E)}\right)\right) t(E) \\
\cdot & \tilde{A}\left(\gamma^{t(E)}\right)(1+h O(1)),
\end{aligned}
$$

where the sum $\sum_{n} \sum_{\Gamma(E)}$ is over all triples $\left(n, t(E), \gamma^{t}(E)\right)$ such that $\gamma^{t(E)}$ is a periodic orbit of primitive period $t(E)$ and energy $E$, and $n$ is any integer such that $n t(E) \in \operatorname{supp} f$. Here $W_{E}\left(\gamma^{t(E)}\right)$ is Hamilton's principal function, i.e. the Legendre transform of the classical action along $\gamma^{t(E)} \cdot \tilde{m}\left(\gamma^{t(E)}\right)$ is a Maslov index associated with the orbit $\gamma^{t(E)}$, and is given by (5.11). The quantity $\widetilde{A}\left(\gamma^{t(E)}\right)$ is equal to $l$ if $d=1$ and equal to

$$
\tilde{A}\left(\gamma^{t(E)}\right)=\frac{1}{\left|D_{T}\right|^{1 / 2}}\left|\prod_{k=1}^{d-1} \cos ^{2} i \beta_{k}\left(\gamma^{t(E)}\right) t(E)\right|^{-1 / 2}
$$

if $d>1$. If $f$ is such that there exists no periodic orbit of primitive period some $t \in \operatorname{supp} f$, then $\left\langle g_{E}, \theta_{h}\right\rangle=o(h)$ as $h \rightarrow 0$.

Remark. The assumption about the existence of a family of periodic orbits is e.g. satisfied, under our assumptions on the potential $V$, if supp $f$ contains one of the point $\frac{2 \pi}{\lambda_{i}}$, where $\lambda_{i}^{2}$ are the eigenvalues of the matrix $A^{2}$. See [10], p. 169. The assumptions about the dependence on $t$ of $S_{t}\left(\gamma^{t}\right)$ can be made more explicit, using the analyticity of $V$. E.g. for $d=1$ the values of $t$ such that the above family exists form an open set and the set of points where $S_{t}\left(\gamma^{t}\right)$ does not satisfy the assumptions is discrete.

We remark that 


$$
\tilde{A}\left(\gamma^{t(E)}\right)=\frac{1}{\left|D_{T}\right|^{1 / 2}}\left(\sum_{\substack{m_{1}=0 \\ m_{d-1}=0}}^{\infty} \exp \left(\sum_{n=1}^{d-1}\left(m_{k}+\frac{1}{2}\right) \beta_{k}\left(\gamma^{t(E)}\right) t(E)\right) .\right.
$$

We assume now that $f$ is such that $f(n t(E))=f(t(E))$ for all integer $n$ such that $n t(E) \in \operatorname{supp} f$. Then the formula of Theorem 5.1 simplifies to

$$
\begin{aligned}
\left\langle g_{E}, \theta_{h}\right\rangle= & \frac{(2 \pi i h)^{-(d-1) / 2}}{\left|D_{T}\right|^{1 / 2}} \sum_{\Gamma(E)} t(E) f(t(E)) \sum_{n} \exp \left(\frac{i}{h} n\left[W_{E}\left(\gamma^{t(E)}\right)-\frac{\pi}{2} m\left(\gamma^{t(E)}\right) h\right]\right) \\
m_{1}=\ldots=m_{d-1}=0 & \exp \sum_{k=1}^{d-1}\left(m_{k}+\frac{1}{2}\right) \beta_{k}\left(\gamma^{t(E)}\right) n t(E)(1+h O(1)),
\end{aligned}
$$

where the sum $\sum_{n}$ is over all $n$ such that $n t(E) \in \operatorname{supp} f$. Let now $N_{f}(t(E))$ be the largest integer $n$ such that $n t(E) \in \operatorname{supp} f$. Then (5.19) reads

$$
\begin{aligned}
& \left\langle g_{E}, \theta_{h}\right\rangle=\frac{(2 \pi i h)^{-\frac{d-1}{2}}}{\left|D_{T}\right|^{1 / 2}} \sum_{\Gamma(E)} t(E) f(t(E)) \sum_{m_{1}=\ldots=m_{d-1}=0}^{\infty} \sum_{n=1}^{N_{f}(t(E))} \\
& \cdot \exp \left(\frac { i } { h } n \left[W_{E}\left(\gamma^{t(E)}\right)-\frac{\pi}{2} m\left(\gamma^{t}(E)\right) h-\sum_{k=1}^{d-1}\left(m_{k}+\frac{1}{2}\right)\right.\right. \\
& \left.\left.\cdot \beta_{k}\left(\gamma^{t}(E)\right) t(E) h\right]\right)(1+h O(1) .
\end{aligned}
$$

Effectuating this sum we then get

$$
\begin{gathered}
\left\langle g_{E}, \theta_{h}\right\rangle=\frac{(2 \pi i h)^{-(d-1) / 2}}{\left|D_{T}\right|^{1 / 2}} \sum_{\Gamma(E)} t(E) f(t(E)) \sum_{k=1}^{d-1} \sum_{m_{k}=0}^{\infty} \\
\frac{1-\exp \left(\frac{i}{h}\left(N_{f}(t(E))\left[W_{E}-\frac{\pi}{2} m^{\prime} h\right]\right)\right.}{1-\exp \left(\frac{i}{h}\left[W_{E}-\frac{\pi}{2} m^{\prime} h\right]\right.} \\
\cdot \exp \left(\frac{i}{h}\left[W_{E}-\frac{\pi}{2} m^{\prime} h\right]\right)(1+h O(1)),
\end{gathered}
$$

with $m^{\prime} \equiv \frac{2}{\pi} i \sum_{k=1}^{d-1}\left(m_{k}+\frac{1}{2}\right) \beta_{k} t(E)+\tilde{m}\left(\gamma^{t(E)}\right)$. The quantity on the left hand side can also be written, using (5.1) in the form

$$
\left\langle\exp \left(\frac{i}{h} E t\right) f(t), \theta_{h}(t)\right\rangle=\sum_{n=1}^{\infty} \int \exp \left(-\frac{i}{h} t\left(\lambda_{n}(h)-E\right)\right) f(t) d t \equiv \operatorname{Tr}(\hat{f}(H-E)),
$$

for any $f \in \mathscr{S}\left(\mathbb{R}_{+}\right)$, where $\hat{f}(\lambda) \equiv \int \exp \left(-\frac{i}{h} t \lambda\right) f(t) d t$. $\operatorname{Tr}(\hat{f}(H-E))$ is by definition the middle term in (5.20) whenever it exists.

We can then formulate the above results in the following 
Theorem 5.2. Under the same assumption as in Theorem 5.1 and with $f$ such that $f(n t(E))=f(t(E))$ for all periods $t(E)$ to the energy $E$ and all integers $n$ such that $n t(E) \in \operatorname{supp} f$, then

$$
\begin{aligned}
\langle\exp & \left.\left(\frac{i}{h} E t\right) f(t), \theta_{h}(t)\right\rangle=\operatorname{Tr} \hat{f}(H-E)=(2 \pi i h)^{-(d-1) / 2}\left|D_{T}\right|^{-1 / 2} \sum_{T(E)} f(t(E)) t(E) \\
& \cdot \sum_{k=1}^{d-1} \sum_{m_{k}=0}^{\infty} \exp \left(\frac{i}{h}\left[W_{E}\left(\gamma^{t(E)}\right)-\frac{\pi}{2} m^{\prime}\left(\gamma^{t(E)}\right) h\right]\right) \\
& \cdot\left[1-\exp \left(\frac{i}{h}\left[W_{E}\left(\gamma^{t(E)}\right)-\frac{\pi}{2} m\left(\gamma^{t(E)}\right) h\right]\right)\right]^{-1} \\
& \cdot\left(1-\exp \left(\frac{i}{h} N_{f} t(E)\left[W_{E}\left(\gamma^{t(E)}\right)-\frac{\pi}{2} m^{\prime}\left(\gamma^{t(E)}\right) h\right]\right)(1+h(O(1)),\right.
\end{aligned}
$$

where $\hat{f}$ is the Fourier transform of $f, W_{E}\left(\gamma^{t(E)}\right)$ is Hamilton's principal function, i.e. the Legendre transform of the classical action along the classical orbit $\gamma^{t(E)}$ of period $t(E) . m^{\prime}$ is the sum of an Arnold-Maslov index $m$ associated with $\gamma^{t(E)}$ and the expression

$$
\frac{2}{\pi} \mathrm{ih} \sum_{k=1}^{d-1}\left(m_{k}+\frac{1}{2}\right) \beta_{k} \gamma^{t(E)} t(E)
$$

involving the stability angles $\beta_{k}$ of the Poincare mapping. The sum $\sum_{T^{(E)}}$ is over all periodic orbits of period $t(E)$.

We also note that Theorem 5.1 yields the following, taking $f$ such that its Fourier transform has support containing only a finite number of points of the spectrum of $H$ :

Theorem 5.3. Under the assumption of Theorem 5.2 and assuming moreover that to a given energy $E$ there is only one classical orbit, we have that the eigenvalues of $H$ are given asymptotically as $h \rightarrow 0$ by the values of the energy $E$ satisfying the extended Bohr-Sommerfeld quantization conditions

$$
W_{E}=\oint p(q, E) d q=\oint p(q, t) d q+E t(E)=2 \pi h\left(n+\frac{1}{4} m+\frac{12}{4} \frac{2}{\pi} i \sum_{k=1}^{d-1}\left(m_{k}+\frac{1}{2}\right) \beta_{k} t(E)\right) .
$$

Remark. For a derivation of relative formulae for the corresponding JWKB quantities, see [11], [15], [16], [22], [23], [29].

Remark. We can also consider the generalized function

$$
\zeta(s) \equiv \sum_{n} \frac{1}{n^{s}} \equiv \frac{1}{\Gamma(s)} \int_{0}^{\infty} t^{s-1} \theta_{h}(i t) d t .
$$

Since $\lambda_{n}=O\left(n^{d}\right)$ as $n \rightarrow \infty$ we have that $\zeta(s)$ is defined as an analytic function for $\operatorname{Res}>d$.

For the one-dimensional harmonic oscillator

$$
\zeta(s)=\frac{1}{n^{s}}\left(2^{s}-1\right) \zeta_{R}(s)
$$


with $\zeta_{R}$ the Riemann $\zeta$-function $\zeta_{R}(s)=\sum \frac{1}{n^{s}}$. In the general case $\zeta$ is the $\zeta$-function for the Schrödinger operator $H$ in as much as it has the same relation to the $\theta$-function for the Schrödinger operator as the classical Riemann $\zeta$-function has with the classical theta function, namely $\zeta$ is the Mellin transform of the analytic continuation to imaginary $t$ of the theta function $\theta$. It also coincides with the so-called Minakshisundaram-Plejel function, in the case of manifolds.

From the above results about the theta function $\theta(t)$ and its asymptotic behaviour as $h \rightarrow 0$, one can derive asymptotic expressions for the $\zeta$-function in terms of classical periodic orbits.

Acknowledgements. We are very grateful to Professors Monroe Donsker, Henri Epstein, Ruedi Seiler and Edy Zehnder for very interesting and helpful discussions. We thank the Mathematics Departments of Bielefeld and Oslo Universities, the Physics Department, the USP-Mathematitiserung and ZIF of Bielefeld University, as well as the CNRS-CPT and the University d'Aix-Marseille II for kind invitations. We are very grateful to Mrs. Richter for her patience and a skillful typing of the manuscript.

\section{References}

1. Albeverio, S., Blanchard, Ph., Høegh-Krohn, R.: Oscillatory integrals and the method of stationary phase in infinitely many dimensions II. Preprint 1980. The results have been announced in Blanchard, Ph.: Asymptotic expansions of Fresnel integrals relative to a non-singular quadratic form. L. Streit, (ed.) Quantum fields-Algebras, Processes, pp. 91-106. Wien: Springer 1980

2. Albeverio, S., Hфegh-Krohn, R.: Invent. Math. 40, 59-106 (1977)

3. Albeverio, S., H申egh-Krohn, R.: Mathematical theory of Feynman path integrals, Lecture Notes in Mathematics Vol. 523. Berlin: Springer 1976

4. Albeverio, S., Hoegh-Krohn, R.: Feynman path integrals and the corresponding method of stationary phase, pp. 3-57 in Feynman path Integrals; Proc. Marseille 1978. Lecture Notes in Physics Vol. 106. Berlin: Springer 1979

5. Albeverio, S., Blanchard, Ph., Hфegh-Krohn, R.: The trace formula for the Schrödinger operators, Materialien des Universitätsschwerpunktes Mathematisierung der Einzelwissenschaften, Materialien XXVII, Bielefeld (Febr. 1980)

6. Albeverio, S., Blanchard, Ph., Hфegh-Krohn, R.: Stationary phase for the Feynman integral and trace formula. In: Functional Integration. J. P. Antoine, E. Tirapegui, (eds.) pp. 23-43. New York: Plenum Press (1980)

7. Albeverio, S., Blanchard, Ph., Hфegh-Krohn, R.: Feynman path integrals, the Poisson formula and the theta function for the Schrödinger operators. In: Trends in Applications of Pure Mathematics to Mechanics, Vol. III, pp. 1-22 R. J. Knops (ed.). New York: Pitman 1981

8. Amann, H., Zehnder, E.: Nontrivial solutions for a class of nonresonance problems and applications to nonlinear differential equations. Zürich-Bochum Preprint 1979

9. Baltes, H. P., Hilf, E. R.: Spectra of finite systems, Bibl. Inst. Mannheim 1976

10. Berger, M. S.: Nonlinearity and functional analysis. New York: Academic Press 1977

11. Berry, M. V., Mount, K. E.: Rep. Prog. Phys. 35, 315-397 (1972)

12. Chazarain, J.: Spectre d'un Hamiltonien quantique et mécanique classique. Université de Nice, Preprint

13. Chazarain, J.: Invent. Math. 24, 65-82 (1974)

14. Colin de Verdiére, Y.: Comp. Math. 27, 83-106 (1973); Comp. Math. 27, 159-184 (1973)

15. Dashen, R., Hasslacher, B., Neveu, A.: Phys. Rev. D 10, 4114 (1974)

16. De Witt-Morette, C., Maheshwari, A., Nelson, B.: Phys. Rept. 50, 256-372 (1979)

17. Duistermaat, J. J.: Commun. Pure Appl. Math. 27, 207-281 (1974)

18. Duistermaat, J. J., Guillemin, V.: The spectrum of positive elliptic operators and periodic geodesics. Proc. AMS Summer Inst. Differ. Geom., Stanford, 1973 
19. Eckmann, J. P., Sénéor, R.: Arch. Rat. Mech. Anal. 61, 153-173 (1976)

20. Guillemin, V., Weinstein, A.: Bull. Am. Math. Soc. 82, 92-95 (1976)

21. Guillemin, V., Sternberg, S.: Geometric asymptotics Math. Surv. 14, Providence: A.M.S. (1977)

22. Gutzwiller, M. C.: J. Math. Phys. 8, 1979-2000 (1967); J. Math. Phys. 12 (1971) 343-358

23. Gutzwiller, M. C.: Classical quantization conditions for a dynamical system with stochastic behaviour, pp. 316-325, Stochastic Behaviour in classical and quantum Hamiltonian Systems, Como, 1977, Casati, G., Ford. J. (eds.) Lecture Notes in Physics 93, Berlin, Heidelberg, New York Springer 1979

24. Huber, A.: Math. Ann. 138, 1-26 (1959)

25. Leray, J.: Solutions asymptotiques et groupe symplectique. In: Fourier Integral Operators and Partial Differential Equations Nice 1974, J. Chazarain, (ed.) pp. 73-97 Lecture Notes Mathematics. Berlin: Springer 1975

26. Maslov, V. P.: Théorie des perturbations et méthodes asymptotiques. Trad. J. Lascoux, R. Sénéor, Paris: Dunod-Gauthier-Villars 1972

27. Maslov, V. P.. J. Comp. Math. 1, 744-778 (1961) (transl.)

28. Molchanov, S. A.: Russ. Math. Surv. 30, 1-63 (1975) (transl.)

29. Rajaraman, R.: Phys. Rep. 21C, 227-313 (1975)

30. Selberg, A.: J. Ind. Math. Soc. 20, 47-87 (1956)

31. Sommerfeld, A.: Atombau und Spektrallinien, 8. bzw. 4. Aufl. Braunschweig: Vieweg 1967-69

32. Truman, A.: The polygonal path formulation of the Feyman path integral, In: Feyman Path Integrals, Proc. Marseille 1978. Lecture Notes in Physics Vol. 106, pp. 73-102. Berlin: Springer 1979

33. Voros, A.: Ann. Inst. H. Poincaré 24, 31-50 (1976)

Communicated by J. Ginibre

Received June 9, 1981 